\title{
Effects of the Low-Level Wind Profile on Outflow Position and Near-Surface Vertical Vorticity in Simulated Supercell Thunderstorms
}

\author{
Felicia Guarriello, ${ }^{\mathrm{a}}$ Christopher J. Nowotarski, And Craig C. Epifanio \\ Department of Atmospheric Sciences, Texas A\&M University, College Station, Texas
}

(Manuscript received 6 June 2017, in final form 30 November 2017)

\begin{abstract}
Supercell thunderstorms are simulated using an idealized numerical model to analyze the effects of modifications to the environmental low-level wind profile on near-surface rotation. Specifically, the orientation, magnitude, and depth of the low-level vertical wind shear are modified in several suites of experiments and compared to control simulations with no vertical wind shear in the prescribed layer.

The overall morphology of the simulated supercells is highly sensitive to even shallow changes in the lowlevel wind profile. Moreover, maximum near-surface vertical vorticity varies as the low-level wind profile is modified. The results suggest this is principally a consequence of the degree to which favorable dynamic forcing of negatively buoyant outflow is superimposed upon the near-surface circulation maximum. Simulations with easterly shear and weaker storm-relative winds over the depth of the gust front promote forwardsurging outflow and smaller separation between the near-surface circulation maximum and the mesocyclone aloft compared with other hodograph shapes. This promotes near-surface vertical vorticity intensification in these simulations. Similar trends in near-surface vertical vorticity as a function of low-level shear orientation are observed for varying shear-layer depths and bulk-shear magnitudes over the shear layer. The degree to which specific hodograph shapes promote strong near-surface rotation may vary with different deep-layer wind profiles or thermodynamic environments from those simulated here; however, this study concludes that favorable positioning of the near-surface circulation maximum and mesocyclone aloft are a necessary condition for supercell tornadogenesis and this positioning may be modulated by the low-level wind profile.
\end{abstract}

\section{Introduction}

Supercell thunderstorms, characterized by a persistent rotating updraft or mesocyclone, are among the most dangerous types of convective storms. In addition to other hazards, supercells have drawn significant research attention because of their ability to produce tornadoes (e.g., Browning 1964; Lemon and Doswell 1979; Weisman and Klemp 1986; Bunkers et al. 2000; Markowski et al. 2003a; Coffer and Parker 2015). Indeed, most significant $(\geq \mathrm{EF} 2)$ and violent $(\geq \mathrm{EF} 4)$ tornadoes are produced by supercells (Smith et al. 2012).

Supplemental information related to this paper is available at the Journals Online website: https://doi.org/10.1175/JAS-D-170174.s1.

${ }^{a}$ Current affiliation: KBRwyle, and NOAA, Silver Spring, Maryland.

Corresponding author: Christopher J. Nowotarski, cjnowotarski@ tamu.edu
Despite improvements in forecasting the occurrence of supercell thunderstorms, predicting which supercells will produce tornadoes remains a challenge. The conditions that support supercell thunderstorms, conditional instability in the presence of strong deep-layer vertical wind shear, are often forecast well by operational global models, while higher-resolution convection-allowing models are capable of explicitly resolving supercells. However, a large majority of supercells are not tornadic (e.g., Trapp et al. 2005), and explicit resolution of tornadoes is currently beyond the reach of operational numerical forecasting models. Even radar identification of tornadoes is difficult, considering that many storms with detected low-level rotation are not in fact tornadic (Trapp et al. 2005). A better understanding of why some supercells produce tornadoes while others do not, as well as how this distinction depends on currently predictable environmental parameters, thus remains critical to improving tornado forecasting and advance warnings.

Two environmental parameters in particular, lowlevel shear (herein, low-level refers to heights $<1 \mathrm{~km}$ 
AGL) and lifting condensation level (LCL), have drawn attention in the literature as particularly effective discriminators between nontornadic and tornadic supercells (Rasmussen and Blanchard 1998; Thompson et al. 2003; Craven and Brooks 2004). Though the precise connections between low-level shear, LCL, and supercell tornadogenesis are not fully understood, some straightforward hypotheses have been proposed.

In terms of shear, stronger low-level shear is often associated with larger storm-relative helicity, implying greater potential for updraft rotation through the tilting and stretching of environmental streamwise vorticity within the supercell updraft, which is responsible for the generation of the midlevel mesocyclone (Davies-Jones 1984). In turn, as rotation aloft intensifies, the associated nonlinear dynamic low pressure perturbation deepens, enhancing the upward-directed perturbation pressure gradient force below the vorticity maximum. Near-ground rotation is generally considered to develop through a separate mechanism relying on the reorientation of baroclinically generated horizontal vorticity in low-level downdrafts within supercell outflow (Markowski and Richardson 2009). Thus, one hypothesized dynamical explanation for the link between low-level shear and supercell tornadoes is an increased dynamic updraft forcing, or "suction," within the negatively buoyant near-surface cold pool air, where the requisite circulation for tornadoes is produced within supercells (Markowski and Richardson 2009, 2014). This forcing has the effect of both drawing circulation-rich air into the updraft and amplifying the associated vertical vorticity through stretching. A considerable body of work exists regarding the processes that produce the pretornadic circulation at the ground within supercell thunderstorms; however, this study is focused primarily on the factors that promote intensification of this rotation to tornado strength.

Dynamic updraft forcing and stretching are likely necessary to amplify the near-ground vorticity to tornado strength. That said, the effectiveness of the stretching depends on the degree to which the circulation-rich outflow air can be lifted, which is in turn influenced by the low-level relative humidity, often expressed in terms of LCL. A higher LCL implies greater latent cooling below the cloud base associated with hydrometeor phase changes and more negatively buoyant outflow, which then modulates the effectiveness of the low-level dynamic lifting and subsequent vorticity stretching. Forecasters, storm chasers, and researchers have often observed that so-called outflowdominant storms are less likely to produce tornadoes, with both observational studies (e.g., Markowski et al. 2002; Grzych et al. 2007; Hirth et al. 2008) and numerical simulation studies (Brooks et al. 1994; Gilmore and
Wicker 1998; Snook and Xue 2008; Markowski and Richardson 2014, 2017) supporting the idea that overly cold or surging outflow is detrimental to low-level rotation and tornadoes in supercell thunderstorms. One explanation is the hypothesis that cold, more negatively buoyant outflow is likely to be more resistant to dynamic updraft forcing and subsequent low-level vorticity intensification through stretching (e.g., Leslie and Smith 1978; Markowski et al. 2003b; Markowski and Richardson 2009, 2014, 2017).

Whereas many of the current lines of thinking and research efforts have been directed toward the aforementioned hypothetical relationship between low-level shear, LCL, and supercell tornadoes, in this paper, we test an alternative (though related) hypothesis that has gained less attention in the literature. The leading edge of overly cold outflow is also more likely to surge forward, undercutting the overlying midlevel updraft, perhaps leading to a mismatch in the positioning of nearground circulation and favorable dynamic updraft forcing supplied by the mesocyclone aloft (e.g., Snook and Xue 2008; Markowski and Richardson 2014). Observations suggest that supercell tornadoes may become more cyclic if the advection of the tornado does not match that of the overlying updraft (Dowell and Bluestein 2002). Moreover, using dual-Doppler analyses, Marquis et al. (2012) observed that tornado longevity was increased for a tornado that maintained a position beneath the midlevel updraft relative to a case where the tornado dissipated when the updraft became tilted and the tornado was displaced from favorable convergence. Other visual (e.g., Wakimoto and Martner 1992) and radar (e.g., Skinner et al. 2014) observations support the notion that tornado development and maintenance depends on favorable alignment between the low-level circulation/tornado and the overlying mesocyclone. Several numerical simulation studies have also found that outflow surging ahead of the mesocyclone aloft leads to an unfavorable alignment for strong near-ground rotation (e.g., Brooks et al. 1994; Snook and Xue 2008; Markowski and Richardson 2014).

Building on such studies, we test the hypothesis that while dynamic updraft forcing and outflow buoyancy are clearly important, these factors are irrelevant if the favorable dynamic forcing provided by the mesocyclone aloft is not properly aligned with the near-ground circulation. The low-level wind profile (both the magnitude of the storm-relative winds and the shear perpendicular to the gust front) and the LCL (through its modulation of outflow buoyancy) are expected to have important impacts on this alignment, because of their effects on the motion and positioning of the cold pool and its associated near-surface pretornadic vertical vorticity. For the 
present study, we focus on the impact of the low-level wind profile on the alignment, while the role of the LCL will be explored in future work.

Support for these hypotheses and guidance for our experiment design come from recent studies suggesting that particular features of the low-level wind profile, including the direction of the low-level shear vector and/or storm-relative winds over even shallower layers than $1 \mathrm{~km}$, are relevant in discriminating between nontornadic and tornadic supercells. Observations of the so-called sickle low-level hodograph (Miller 2006; Esterheld and Guiliano 2008; Kis and Straka 2010) suggest hodographs with strong, predominately streamwise environmental horizontal vorticity near the ground are favorable for tornadoes. Despite similar overall storm-relative helicity and deep-layer shear, hodographs with significant streamwise vorticity and southerly shear in the lowest $500 \mathrm{~m}$ were more likely to result in tornadic storms than those with a dominant crosswise component of vorticity and easterly shear in a study of proximity soundings (Nowotarski and Jensen 2013). Similar hodograph differences were observed during the VORTEX2 field project (Parker 2014). Recent numerical modeling studies suggest that the orientation of the low-level shear vector may also control the position of near-ground circulations relative to the mesocyclone aloft, with Coffer and Parker (2017) finding that a wind profile with crosswise vorticity led to transient near-ground circulations that were typically located rearward of the mesocyclone aloft.

Though primarily limited to interactions between the ambient wind profile and cold pools in the context of squall lines, prior studies have determined that, in addition to the cold pool intensity (its buoyancy relative to the environment and its depth), cold pool propagation is dependent on the ambient wind profile. Idealized studies indicate that density currents are generally shallower and faster moving with no shear-oriented normal to the density current or with a component of the shear vector along the direction of its motion (e.g., Xu 1992; Xue 2000). Rotunno et al. (1988) suggest that conditions for intense squall-line updrafts are maximized when horizontal vorticity generated along the leading edge of outflow (i.e., the gust front) is balanced by environmental horizontal vorticity parallel to the gust front but oriented in the opposite direction. In their simulations, if the vorticity associated with a strong cold pool was not balanced by that associated with environmental vertical shear directed perpendicular to the gust front in the direction of its motion or the shear opposes (i.e., points toward) the gust front, outflow advanced ahead of the overlying convection, and ambient air was lifted rearward over the cold pool. Weisman and Klemp $(1982,1984)$ found that gust fronts are less likely to undercut storms as vertical wind shear in the environment increases and that an intermediate range of vertical wind shear exists where the development of lowlevel vertical vorticity was most favored. Houston (2016) examined the interaction between ambient wind shear and overlying convection in two-dimensional simulations, finding that low-level lifting along the leading edge of outflow increased and that phasing between the gust front and an overlying updraft improved as the amount of shear normal to (pointed in the direction of) the gust-front motion increased.

Snook and Xue (2008) concluded that "supercell tornadogenesis likely requires an optimal balance between cold pool strength and environmental flow." Supercell outflow is inherently more three-dimensional and often less steady than that of a squall line, but interactions between the wind profile and cold pool may influence the alignment of the near-ground circulation and overlying mesocyclone, thereby modulating lowlevel ascent. Results from his experiments led Houston (2016) to speculate that in localized regions of a supercell gust front, the shear orientation might enhance lowlevel lifting. Though lifting driven by gust-front-shear interactions may be relevant, our study is primarily focused on how the low-level wind profile modulates the position of low-level outflow and embedded circulations relative to the overlying mesocyclone and presumably attendant regions of favorable dynamically induced, upward vertical perturbation pressure gradient force. For instance, a typical supercell moving from west to east might be characterized by a rear-flank gust front oriented from southwest to northeast, such that an increase in easterly (westerly) shear may be expected to cause the outflow and attendant near-surface circulation to advance more (less) relative to the midlevel mesocyclone. Indeed, Frame and Markowski (2013) found in numerical simulations including anvil shading that decreased mixing in the inflow beneath the anvil cloud led to a longer easterly shear vector, which encouraged outflow to undercut the updraft, reducing the magnitude of near-surface rotation.

The present study tests the relationship between the low-level wind profile and the relative positioning between the near-surface circulation and the dynamic forcing aloft, as explored in a set of idealized simulations of supercells. Experiments are conducted in which the magnitude, direction, and depth of the low-level shear profile are systematically varied, with the thermodynamic profile held constant. Among the questions to be addressed are the following:

- How do the direction, depth, and magnitude of the low-level shear and storm-relative winds affect the ultimate strength of near-surface rotation? 
- Do changes in the low-level wind profile affect the properties and/or the positioning of the supercell outflow relative to the midlevel mesocyclone and the most favorable dynamic lifting?

- Does this relative positioning of outflow and the midlevel mesocyclone influence the strength of nearsurface rotation?

The following section describes the design of the experiment, including the model configuration and range of initial conditions. Section 3 presents the simulation results, including discussion of their relevance in the context of other hypotheses regarding supercell tornadoes, and a summary with concluding remarks is offered in the final section.

\section{Methods and experiment design}

Several suites of idealized, horizontally homogeneous numerical simulations were performed to investigate the sensitivity of simulated supercell thunderstorms to modifications to the low-level wind profile. Each suite contains systematic alterations to the low-level wind profile so as to investigate a parameter space of lowlevel shear-vector orientations and magnitudes over a range of depths.

\section{a. Numerical model configuration}

All simulations are performed using Cloud Model 1 (CM1), release 17 (Bryan and Fritsch 2002; Bryan 2002). CM1 is a three-dimensional, nonhydrostatic, fully compressible, time-dependent numerical model designed for idealized studies of atmospheric phenomena. The horizontal grid spacing is $500 \mathrm{~m}$, with a stretched vertical grid with $50-\mathrm{m}$ spacing below $4 \mathrm{~km}$, increasing to $500-\mathrm{m}$ grid spacing above $15 \mathrm{~km}$. The horizontal domain spans $175 \mathrm{~km} \times 175 \mathrm{~km}$ in the horizontal up to a height of $20 \mathrm{~km}$. The upper and lower boundary conditions are rigid and free slip with open radiative lateral boundaries and a Rayleigh-damping sponge layer above $15 \mathrm{~km}$. Each simulation is run for $3 \mathrm{~h}$ with adaptive time stepping (the large time step is adaptively modified throughout the simulation to maintain computational stability) using an initial large time step of $5 \mathrm{~s}$. The Morrison double-moment scheme (Morrison et al. 2005) is used to parameterize cloud microphysics. Sub-grid-scale turbulence is parameterized with a 1.5-order closure (Deardorff 1980). The Coriolis force, radiation, and surface fluxes of heat, moisture, and momentum are not included.

\section{b. Base states and initialization}

For all simulations, the prescribed thermodynamic and kinematic vertical profile is applied over the entire

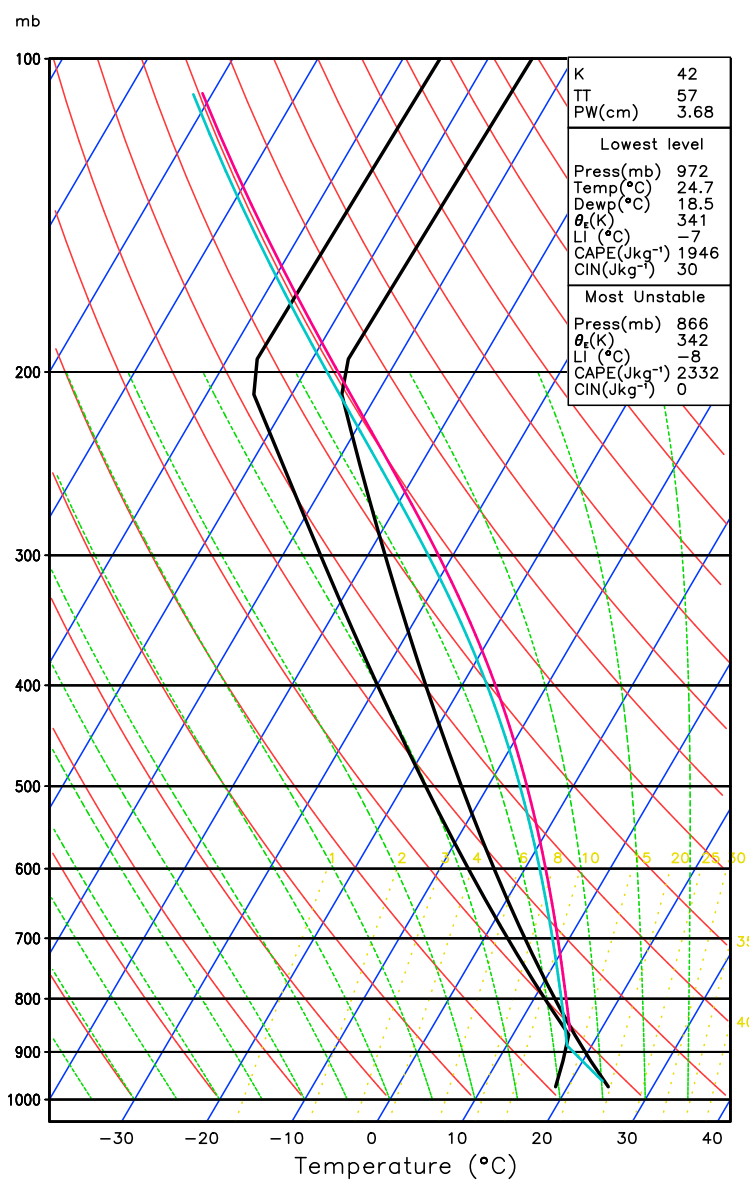

FIG. 1. Skew $T-\log p$ diagram of the thermodynamic base state for all simulations. Parcel paths are shown for a surface-based (cyan) and most unstable (magenta) parcel.

domain such that every base state is horizontally homogeneous. The simulations are all initialized from the same thermodynamic profile, defined as an idealized analytic sounding supportive of deep moist convection as is commonly used in idealized studies of supercell thunderstorms (Fig. 1; Weisman and Klemp 1982). The base-state wind profile for each simulation is derived from an idealized quarter-circle, clockwise-turning hodograph (Weisman and Klemp 1984) with a turning shear vector over the lowest $2 \mathrm{~km}$, a unidirectional shear of $15 \mathrm{~m} \mathrm{~s}^{-1}$ from 2 to $6 \mathrm{~km}$, and constant winds above $6 \mathrm{~km}$ (the black curve in Fig. 2).

Modifications to the lower levels of the wind profile are made in each experiment as follows and summarized in Table 1. Each suite of experiments is composed of simulations in which the low-level wind profile is modified over a layer of depth $d$ immediately above the ground. For a given depth, four simulations are computed: three in which the winds over the layer are defined as a constant shear profile, with shear oriented at 


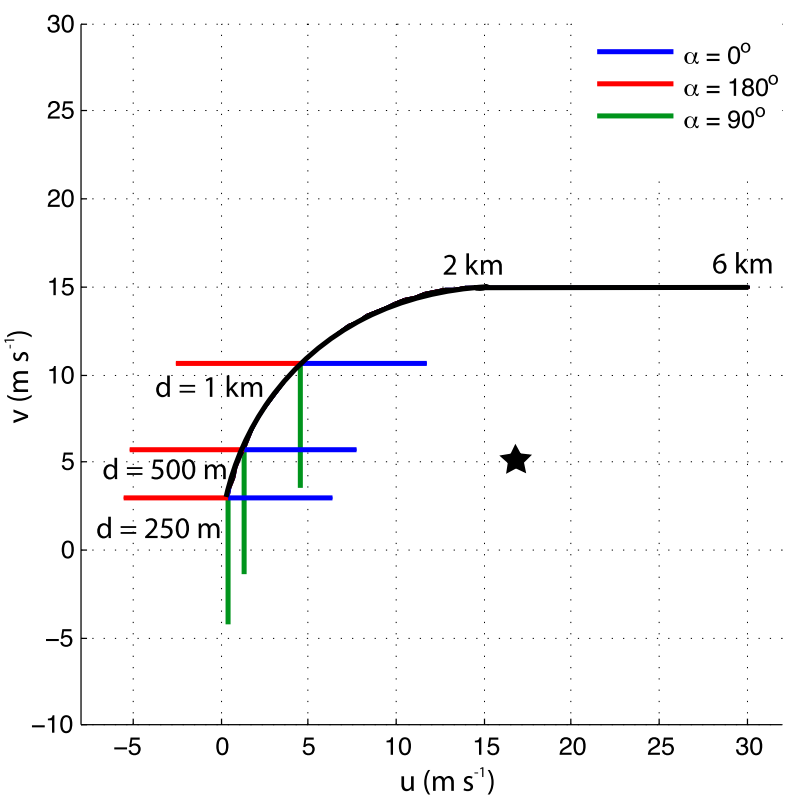

FIG. 2. Hodographs showing kinematic base states for each experiment where $7 \mathrm{~m} \mathrm{~s}^{-1}$ of bulk shear is distributed over varying depths $d$ with varying angles $\alpha$ with respect to the deep-layer hodograph (black). The average storm motion over the last hour of all the simulations, calculated by tracking the $1-6-\mathrm{km}$ UH maximum, is shown as a black star.

varying angles $\alpha$, and a fourth control experiment in which the winds below height $d$ are constant such that there is no vertical wind shear in this layer (Fig. 2). The values of $\alpha$ are defined based on the deviation of the shear-vector orientation from that of an easterly surface wind [comparable but not identical to the critical angle defined by Esterheld and Guiliano (2008)] with three values: $\alpha=0^{\circ}$ (easterly shear), $\alpha=90^{\circ}$ (southerly shear), and $\alpha=180^{\circ}$ (westerly shear). Hodographs for suites of simulations with $d=250 \mathrm{~m}, 500 \mathrm{~m}$, and $1 \mathrm{~km}$ are shown in Fig. 2. The magnitude of the bulk shear over layer $d$ is held constant at $7 \mathrm{~m} \mathrm{~s}^{-1}$, though a second suite of $d=$ $500 \mathrm{~m}$ simulations were performed with a bulk shear of $15 \mathrm{~m} \mathrm{~s}^{-1}$ to test the sensitivity to shear magnitude.
Ideally, to isolate the role of low-level winds on outflow and near-surface circulation position relative to favorable dynamic lifting provided by the mesocyclone aloft, streamwise vorticity in the environment [as quantified by storm-relative helicity (SRH)] should be held constant between simulations. We have designed the wind profile modifications to minimize variations in SRH in experiment suites with similar $d$, but some differences in SRH are unavoidable. SRH values for each simulation are listed in Table 2. SRH generally decreases for each $\alpha$ as $d$ increases, whereas for a given $d$, cases where $\alpha=90^{\circ}$ have the greatest SRH. Thus, there is some potential that differences in near-surface rotation exist because of changes in the intensity of the lowand midlevel mesocyclone (and the attendant dynamic updraft forcing) as SRH increases in addition to the positional effects that are the focus of our investigation. Though the results suggest that this alternative mechanism is outweighed by positional effects in these simulations, implications of these variations in SRH are considered when interpreting our results.

It should also be noted that changes in the shearvector orientation also unavoidably lead to changes in the magnitude of the storm-relative wind opposing the gust front, such that separating effects due strictly to the shear orientation from those due to the strength of the low-level storm-relative winds is difficult. The components of the $0-1-\mathrm{km}$ average storm-relative wind and the $0-1-\mathrm{km}$ bulk-shear vector that oppose the simulated gust fronts, calculated from the base state of each simulation, are shown in Table 3. These are defined as the components of each vector that are perpendicular to the average gust-front position over the last $2 \mathrm{~h}$ of each simulation, with the convention that positive (negative) values are pointed toward (away from) the gust front. The average gust-front position is obtained by averaging the potential temperature perturbation at the lowest grid level over the last $2 \mathrm{~h}$ of each simulation on a $20 \mathrm{~km} \times 20 \mathrm{~km}$ subdomain centered on the $1-6-\mathrm{km}$ integrated updraft helicity maximum at each 5-min output

TABLE 1 . Simulation names as determined by $d, \alpha$, and bulk low-level shear magnitude.

\begin{tabular}{llll}
\hline & \multicolumn{1}{c}{$d=250 \mathrm{~m}$} & \multicolumn{1}{c}{$d=500 \mathrm{~m}$} & $d=1 \mathrm{~km}$ \\
\hline Control & CTL-250M & CTL-500M & CTL-1KM \\
Bulk shear $=7 \mathrm{~m} \mathrm{~s}^{-1}$ & 0DEG-250M-7MS & 0DEG-500M-7MS & 0DEG-1KM-7MS \\
$\begin{array}{l}\alpha=0^{\circ} \\
\alpha=90^{\circ}\end{array}$ & 90DEG-250M-7MS & 90DEG-500M-7MS & 90DEG-1KM-7MS \\
$\alpha=180^{\circ}$ & 180DEG-250M-7MS & 180DEG-500M-7MS & 180DEG-1KM-7MS \\
Bulk shear $=15 \mathrm{~m} \mathrm{~s}^{-1}$ & - & 0DEG-500M-15MS & - \\
$\alpha=0^{\circ}$ & - & 90DEG-500M-15MS & - \\
$\alpha=90^{\circ}$ & - & 180DEG-500M-15MS & - \\
\hline
\end{tabular}


TABLE 2. Base-state 0-3-km (SRH3), 0-1-km (SRH1) and 0-500-m (SRH0.5) SRH $\left(\mathrm{m}^{2} \mathrm{~s}^{-2}\right.$ ) for each control, $7 \mathrm{~m} \mathrm{~s}^{-1}$ bulkshear, and $15 \mathrm{~m} \mathrm{~s}^{-1}$ bulk-shear experiment simulation. SRH calculations are based on storm-relative winds computed from average simulated storm motion over the last hour of each simulation.

\begin{tabular}{|c|c|c|c|c|}
\hline & $\frac{d=250 \mathrm{~m}}{7 \mathrm{~m} \mathrm{~s}^{-1}}$ & $\frac{d=500 \mathrm{~m}}{7 \mathrm{~m} \mathrm{~s}^{-1}}$ & $\frac{d=1 \mathrm{~km}}{7 \mathrm{~m} \mathrm{~s}^{-1}}$ & $\frac{d=500 \mathrm{~m}}{15 \mathrm{~m} \mathrm{~s}^{-1}}$ \\
\hline \multicolumn{5}{|l|}{ SRH3 } \\
\hline Control & 291 & 247 & 168 & 247 \\
\hline$\alpha=0^{\circ}$ & 304 & 242 & 128 & 236 \\
\hline$\alpha=90^{\circ}$ & 407 & 357 & 254 & 477 \\
\hline$\alpha=180^{\circ}$ & 279 & 252 & 207 & 258 \\
\hline \multicolumn{5}{|l|}{ SRH1 } \\
\hline Control & 124 & 79 & 0 & 79 \\
\hline$\alpha=0^{\circ}$ & 136 & 76 & -40 & 68 \\
\hline$\alpha=90^{\circ}$ & 239 & 190 & 86 & 310 \\
\hline$\alpha=180^{\circ}$ & 111 & 84 & 40 & 90 \\
\hline \multicolumn{5}{|l|}{ SRH0.5 } \\
\hline Control & 44 & 0 & 0 & 0 \\
\hline$\alpha=0^{\circ}$ & 56 & -4.8 & -20 & -11 \\
\hline$\alpha=90^{\circ}$ & 160 & 110 & 47 & 230 \\
\hline$\alpha=180^{\circ}$ & 32 & 5 & 20 & 11 \\
\hline
\end{tabular}

interval. The slope of a best-fit line of grid points where the potential temperature perturbation is between -1.1 and $-0.9 \mathrm{~K}$ is used to compute the gust-front orientation angle (relative to a north-south orientation angle of $0^{\circ}$ and an east-west orientation angle of $90^{\circ}$ ) in Table 3 , which is required for the coordinate transformation applied in obtaining the opposing storm-relative winds and shear.

Deep convection is triggered in each simulation using a warm bubble with a horizontal radius of $10 \mathrm{~km}$ and vertical radius of $1400 \mathrm{~m}$ centered $1400 \mathrm{~m}$ above the lower boundary with a maximum potential temperature perturbation of $1 \mathrm{~K}$. Simulations are integrated for $3 \mathrm{~h}$. After the first hour from model initialization, deep convection takes on supercellular characteristics with an established cold pool in every simulation. Thus, our analysis is limited to the last $2 \mathrm{~h}$ of the simulations for the dominant, right-moving supercell in each simulation.

\section{Results and discussion}

\section{a. Influence of storm-relative wind and shear-vector orientation}

Figure 3 shows the simulated storm structure for the experiments with shear-layer depth of $500 \mathrm{~m}$ and bulk shear of $7 \mathrm{~m} \mathrm{~s}^{-1}$, as illustrated in terms of the simulated reflectivity, gust-front position, and the position of maximum vertical vorticity at the surface, along with the updraft velocity at midlevels and region of largest updraft helicity. All fields are shown at the time of largest vertical vorticity at the surface within the updraftrelative windows shown in Fig. 4. Maxima outside this
TABLE 3. Average gust-front orientation angle (relative to a north-south orientation), component of the $0-1-\mathrm{km}$ average storm-relative wind opposing the gust front and component of the $0-1-\mathrm{km}$ shear opposing the gust front for each simulation.

\begin{tabular}{|c|c|c|c|c|}
\hline & $\frac{d=250 \mathrm{~m}}{7 \mathrm{~m} \mathrm{~s}^{-1}}$ & $\frac{d=500 \mathrm{~m}}{7 \mathrm{~m} \mathrm{~s}^{-1}}$ & $\frac{d=1 \mathrm{~km}}{7 \mathrm{~m} \mathrm{~s}^{-1}}$ & $\frac{d=500 \mathrm{~m}}{15 \mathrm{~m} \mathrm{~s}^{-1}}$ \\
\hline \multicolumn{5}{|c|}{ Gust-front orientation angle $\left({ }^{\circ}\right)$} \\
\hline Control & 51 & 53 & 44 & 53 \\
\hline$\alpha=0^{\circ}$ & 47 & 60 & 48 & 44 \\
\hline$\alpha=90^{\circ}$ & 35 & 46 & 49 & 17 \\
\hline$\alpha=180^{\circ}$ & 55 & 62 & 56 & 53 \\
\hline \multicolumn{5}{|c|}{ Storm-relative wind opposing gust front $\left(\mathrm{m} \mathrm{s}^{-1}\right)$} \\
\hline Control & $10.1^{1}$ & 10.3 & 12.6 & 10.3 \\
\hline$\alpha=0^{\circ}$ & 10.4 & 8.4 & 9.9 & 9.2 \\
\hline$\alpha=90^{\circ}$ & 12.4 & 10.3 & 9.6 & 13.6 \\
\hline$\alpha=180^{\circ}$ & 9.8 & 9.4 & 13.4 & 12.6 \\
\hline \multicolumn{5}{|c|}{$0-1-\mathrm{km}$ bulk shear opposing gust front $\left(\mathrm{m} \mathrm{s}^{-1}\right)$} \\
\hline Control & 3.4 & 1.9 & 0.0 & 1.9 \\
\hline$\alpha=0^{\circ}$ & 6.4 & 5.6 & 4.5 & 11.3 \\
\hline$\alpha=90^{\circ}$ & 4.6 & 6.2 & 5.1 & 2.4 \\
\hline$\alpha=180^{\circ}$ & 0.9 & -0.2 & -3.8 & -6.7 \\
\hline
\end{tabular}

window are not expected to be dynamically relevant to tornadogenesis. Though these are snapshots of each storm, the general storm structure is representative of the mature storm morphology in each case (animations of each 500M-7MS simulation are included in the supplemental figures). The 180DEG-500M-7MS supercell often displays a more zonally oriented gust front than the other simulations (cf. Table 3 ) with an elongated reflectivity structure. In contrast, the 90DEG-500M$7 \mathrm{MS}$ supercell often has the most meridionally oriented gust front, and the 0DEG-500M-7MS supercell is characterized by a more occluded structure with a pronounced hook echo and a horseshoe-shaped updraft. This updraft shape is a sustained feature throughout the last $90 \mathrm{~min}$ of the simulation and is unique to the 0DEG500M-7MS simulation, as can be seen in the animations in supplemental Fig. S2. In general, contrasting westerly shear to easterly shear, the $0-1-\mathrm{km}$ shear (storm-relative wind) opposing the gust front increases (decreases) as shown in Table 3 , and the leading edge of the outflow tends to surge to a more forward (with respect to the midlevel updraft), meridionally oriented position.

Along with changes in outflow morphology, the timing of the near-ground vertical vorticity maximum and its position relative to the mesocyclone aloft are affected by the shear-vector orientation. The 0DEG-500M-7MS simulation has its maximum near-surface vertical vorticity at the earliest time of $7800 \mathrm{~s}\left(\zeta=0.06 \mathrm{~s}^{-1}\right.$; Fig. 3b), with 90DEG-500M-7MS following at $9900 \mathrm{~s}(\zeta=$ $0.02 \mathrm{~s}^{-1}$; Fig. 3c). Both CTL-500M and 180DEG-500M$7 \mathrm{MS}$ do not achieve their maximum vertical vorticity $\left(\zeta<0.02 \mathrm{~s}^{-1}\right)$ until the end of the simulations $(10800 \mathrm{~s})$. 

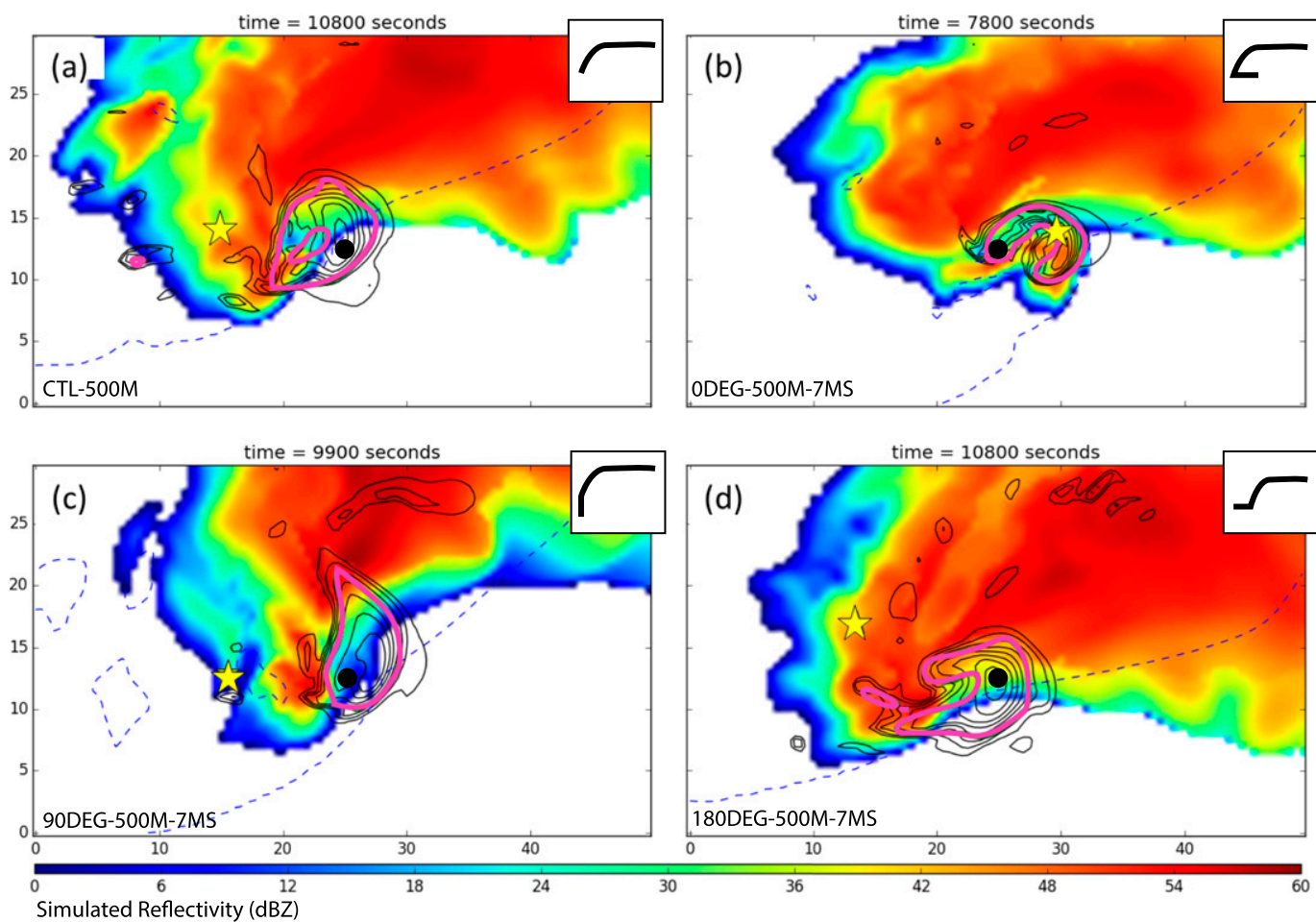

FIG. 3. Simulated reflectivity at the lowest grid level (shaded; dBZ) for (a) CTL-500M, (b) 0DEG-500M-7MS, (c) $90 \mathrm{DEG}-500 \mathrm{M}-7 \mathrm{MS}$, and (d) $180 \mathrm{DEG}-500 \mathrm{M}-7 \mathrm{MS}$ at the time of maximum vertical vorticity at the lowest grid level. The dashed blue line is the $-1-\mathrm{K}$ potential temperature contour at the lowest grid level, black contours are midlevel (4 km AGL) vertical velocity plotted every $5 \mathrm{~m} \mathrm{~s}^{-1}$, the solid magenta contour is the $600 \mathrm{~m}^{2} \mathrm{~s}^{-2} \mathrm{UH}$ outline, the black dot is the maximum integrated $1-6-\mathrm{km} \mathrm{UH}$, and the yellow star is the maximum vertical vorticity at the lowest grid level. Grid-scale labels are in kilometers. Schematics of hodograph shape are shown for reference.

In both CTL-500M and 180DEG-500M-7MS, the leading edge of the outflow, or gust front, is largely restrained behind (i.e., west of) the midlevel mesocyclone. By contrast, even as early as $7800 \mathrm{~s}$, the outflow and maximum near-surface vertical vorticity (yellow star in Fig. 3b) within the cold pool are positioned below the midlevel mesocyclone in the 0DEG-500M-7MS case, perhaps explaining the earlier near-surface vertical vorticity maximum in this simulation. In fact, this is the only case in this suite of simulations where the nearsurface vertical vorticity is ever ahead of the midlevel updraft helicity (UH) maximum (black dot in Fig. 3b).

To assess the degree to which near-surface rotation is located in a position presumably favorable for intensification via dynamic lifting, circulation around a horizontal 2-km square circuit centered on each grid point is computed and plotted in Fig. 4 for the $d=500-\mathrm{m}$ subset of simulations. According to Stokes' theorem, for a horizontal circuit, circulation is equal to the integral of vertical vorticity over the area bounded by the circuit, thus giving an integrated measure of vertical vorticity in the vicinity of each grid point. As discussed by Markowski and Richardson (2014), circulation in this context is a convenient metric for the broad area of rotation available to be intensified by subsequent vorticity stretching (although local vorticity stretching may often increase circulation values as well). In the following analysis, we use the maximum near-surface circulation in these plots as a proxy for the location of the nearsurface mesocyclone.

The distribution of near-surface circulation varies considerably among the $d=500-\mathrm{m}$ simulations (Fig. 4). In all storms, areas of both positive and negative circulation are mostly limited to the outflow of the storm; however, both maximum and minimum values are more intense in the 0DEG-500M-7MS (Fig. 4b) and 90DEG500M-7MS (Fig. 4c) simulations than in the CTL-500M and 180DEG-500M-7MS simulations. The 0DEG500M-7MS case has the greatest maximum near-surface circulation (at this and all times) of any simulation. Moreover, the near-surface circulation maximum is located beneath an area of appreciable 1-6-km integrated updraft helicity $\left(>600 \mathrm{~m}^{2} \mathrm{~s}^{-2}\right)$ indicative of the midlevel mesocyclone, despite an apparently smaller midlevel mesocyclone in this case. A similarly strong area of negative (anticyclonic) circulation is located south of the 

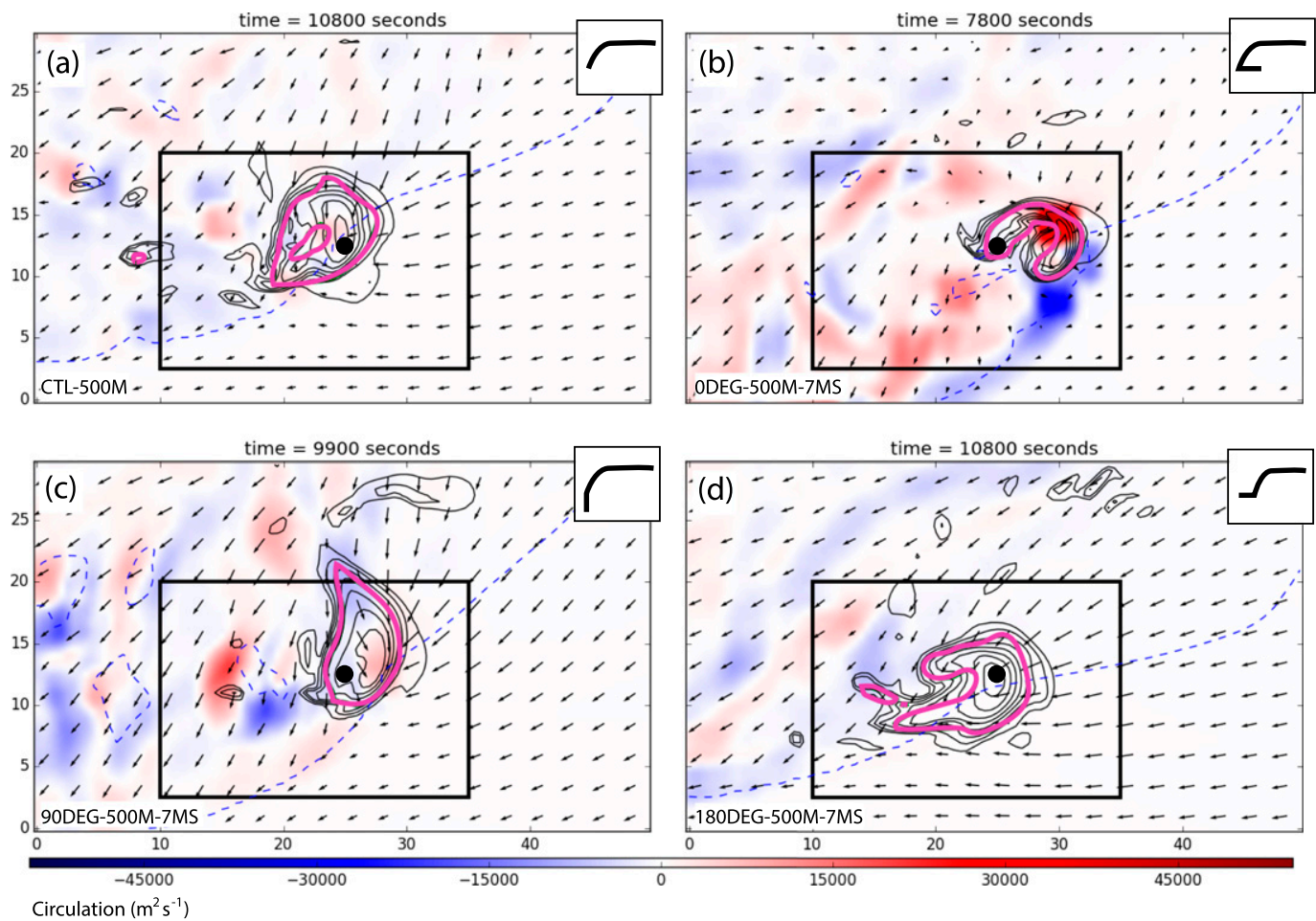

FIG. 4. Circulation computed about 2-km horizontal squares centered on each grid point at the lowest grid level (shaded; $\mathrm{m}^{2} \mathrm{~s}^{-1}$ ) for (a) CTL-500M, (b) 0DEG-500M-7MS, (c) 90DEG-500M-7MS, and (d) 180DEG-500M-7MS at the time of maximum vertical vorticity at the lowest grid level. The dashed blue line is the $-1-\mathrm{K}$ potential temperature contour at the lowest grid level, black contours are midlevel (4 km AGL) vertical velocity plotted every $5 \mathrm{~m} \mathrm{~s}^{-1}$, the solid magenta contour is the $600 \mathrm{~m}^{2} \mathrm{~s}^{-2} \mathrm{UH}$ outline, and the black dot is the maximum 1-6-km integrated UH. Storm-relative winds are plotted as vectors. Grid-scale labels are in kilometers. Schematics of hodograph shape are shown for reference. Black boxes show the region used for constraining the calculation of distance between the midlevel UH maximum and the near-ground circulation maximum as described in the text.

midlevel mesocyclone, indicative of the couplet of positive and negative vertical vorticity often associated with arching vortex lines (e.g., Markowski et al. 2008). A similar, though weaker, circulation couplet exists in the 90DEG-500M-7MS simulation; however, it is located well behind (i.e., west of) the midlevel mesocyclone.

To assess the robustness of the pattern seen above, the distance between the circulation maximum at the lowest grid level (i.e., the near-surface mesocyclone) and the 1-6-km integrated UH maximum (i.e., the mesocyclone aloft) was calculated every $5 \mathrm{~min}(300 \mathrm{~s})$ for each simulation (Fig. 5; blue lines). ${ }^{1}$ This distance (hereafter referred to as the mesocyclone separation distance) is defined as positive (negative) when the near-surface

\footnotetext{
${ }^{1}$ Different definitions of "mesocyclone separation distance" were tested, including using the position of the $z=1-\mathrm{km}$ circulation maximum as the location of the mesocyclone aloft and a larger circuit for circulation calculations. Though small quantitative differences exist, the results are qualitatively similar and appear to be robust across different definitions of this parameter.
}

mesocyclone is west (east) of the mesocyclone aloft. For example, a situation in which the near-surface mesocyclone is located ahead of the overlying storm would have a large negative distance, whereas a situation with a vertically stacked mesocyclone would have a distance approaching zero. There are some unavoidable fluctuations in mesocyclone separation distance, which are a consequence of either competing areas of maximum near-ground circulation or near-ground circulation maxima that exit the search area (dashed blue line segments). Thus, it is important to remember that these time series do not always coherently track a single nearground circulation center; rather, they are based on the instantaneous locations of maximum near-ground circulation and updraft helicity and are better interpreted as the alignment of these quantities at a particular time rather than the motion of discrete vortices over time. Regardless, analysis in later sections of this paper suggests that qualitative interpretations are largely insensitive to these fluctuations. Time series of the maximum near-surface vertical vorticity (red) and 


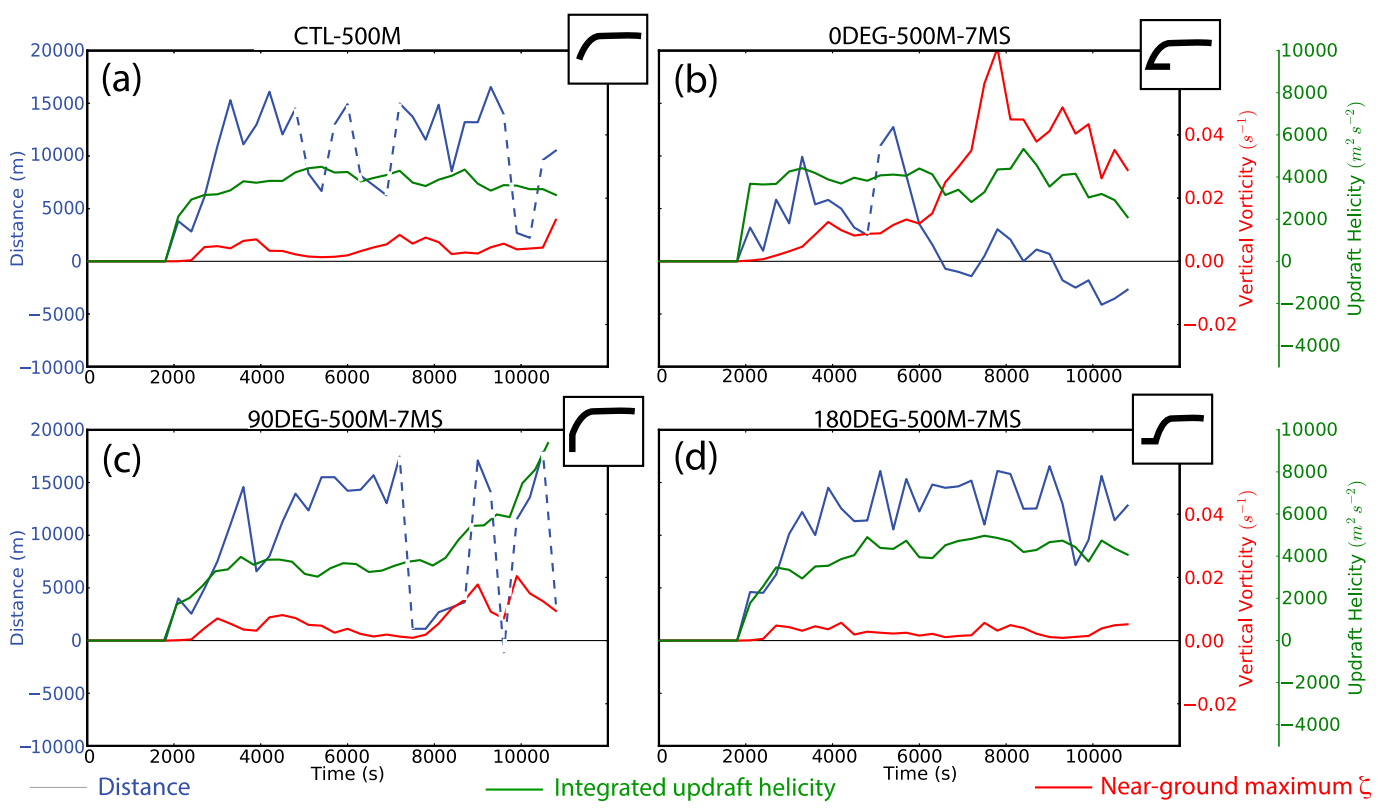

FIG. 5. Time series of the horizontal east-west distance between the locations of the maximum 1-6-km integrated $\mathrm{UH}$ and the maximum low-level circulation (blue), the maximum vertical vorticity at the lowest grid level (red), and the maximum 1-6-km integrated UH (green) for (a) CTL-500M, (b) 0DEG-500M-7MS, (c) 90DEG-500M-7MS, and (d) 180DEG-500M-7MS. Dashed portions of the blue lines indicate time periods when the mesocyclone separation distance algorithm alternates between distinct low-level circulation centers.

maximum UH (green) are also plotted for each simulation.

After the first hour of the simulations, the time series generally support the patterns in Figs. 3 and 4. Overall, the mesocyclone separation distance is generally large and positive for the duration of the CTL-500M and 180DEG-500M-7MS simulations, suggesting that the near-surface circulation maximum is located well west of (behind) the mesocyclone aloft throughout the simulations. The near-surface maximum vertical vorticity remains relatively small $\left(<0.01 \mathrm{~s}^{-1}\right)$ in these simulations. The 90DEG-500M-7MS simulation has generally large mesocyclone separation distance and small nearsurface maximum vertical vorticity except for a slight uptick in vertical vorticity near the end of the simulation concurrent with a decrease in the separation distance (Fig. 5c) as a secondary near-surface circulation closer to the mesocyclone aloft becomes the primary near-surface circulation (the time period between 7000 and $10800 \mathrm{~s}$ in Fig. $5 \mathrm{c}$ is an example of a case where the mesocyclone separation distance algorithm alternates between two competing centers of near-ground circulation). In contrast, the 0DEG-500M-7MS simulation starts with a large positive mesocyclone separation distance that rapidly decreases to near zero and occasionally negative values after $6000 \mathrm{~s}$ (in this case, a single circulation is coherently tracked over time). Soon after, though not immediately concurrent with this decrease in mesocyclone separation, the maximum near-surface vertical vorticity dramatically increases to values consistently over twice as strong as those attained in any of the other simulations. Though not as dramatic, a similar response to decreasing mesocyclone separation distance is suggested intermittently in the other simulations (e.g., around $8000 \mathrm{~s}$ in Fig. 5c and at $10000 \mathrm{~s}$ in Fig. 5a). Updraft helicity, a proxy for mesocyclone intensity, remains fairly consistent around $4000 \mathrm{~m}^{2} \mathrm{~s}^{-2}$ for all of the simulations except for a considerable increase in the 90DEG$500 \mathrm{M}-7 \mathrm{MS}$ simulation after $8000 \mathrm{~s}$. This increase in UH is consistent with the stronger SRH associated with this hodograph.

The $d=500-\mathrm{m}$ simulation results suggest that only in the case with easterly low-level shear and the weakest storm-relative winds opposing the gust front do zones of significant near-surface vertical vorticity in the outflow align with the mesocyclone aloft. In the remaining cases, the circulation-rich outflow air appears mainly restrained behind the mesocyclone by the storm-relative winds. Moreover, the time series suggest a negative correlation between the mesocyclone separation distance and the intensity of near-surface vertical vorticity, with the decrease in separation appearing to lead the vorticity increase in time. These findings are consistent with the hypothesis that intensification of the nearsurface vertical vorticity requires an alignment between the mesocyclone aloft and regions of circulation-rich air 

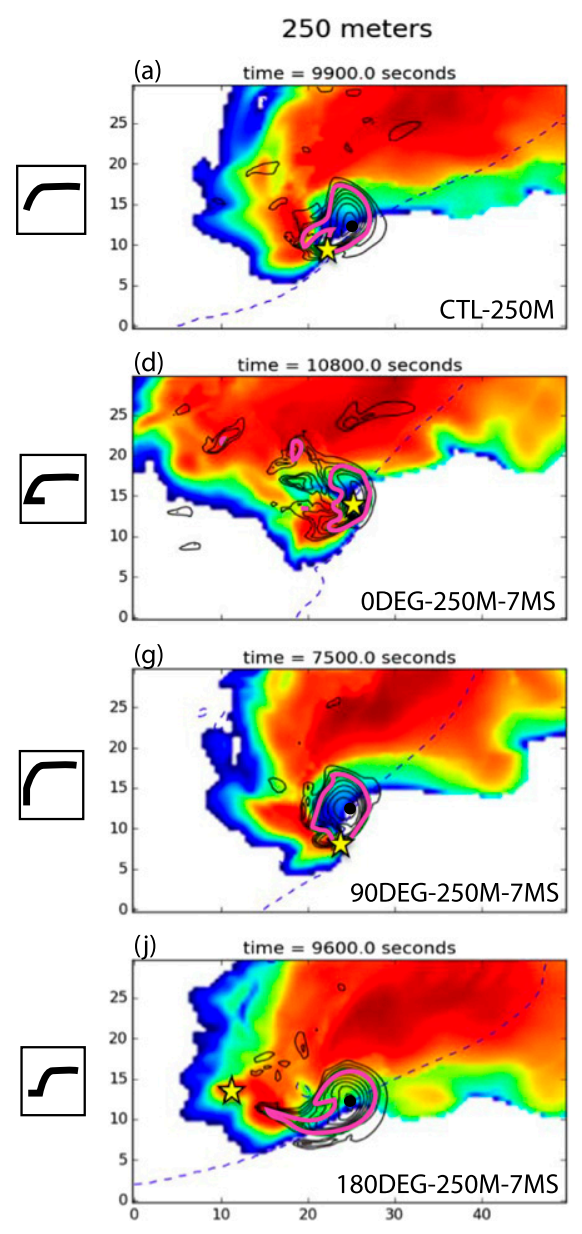
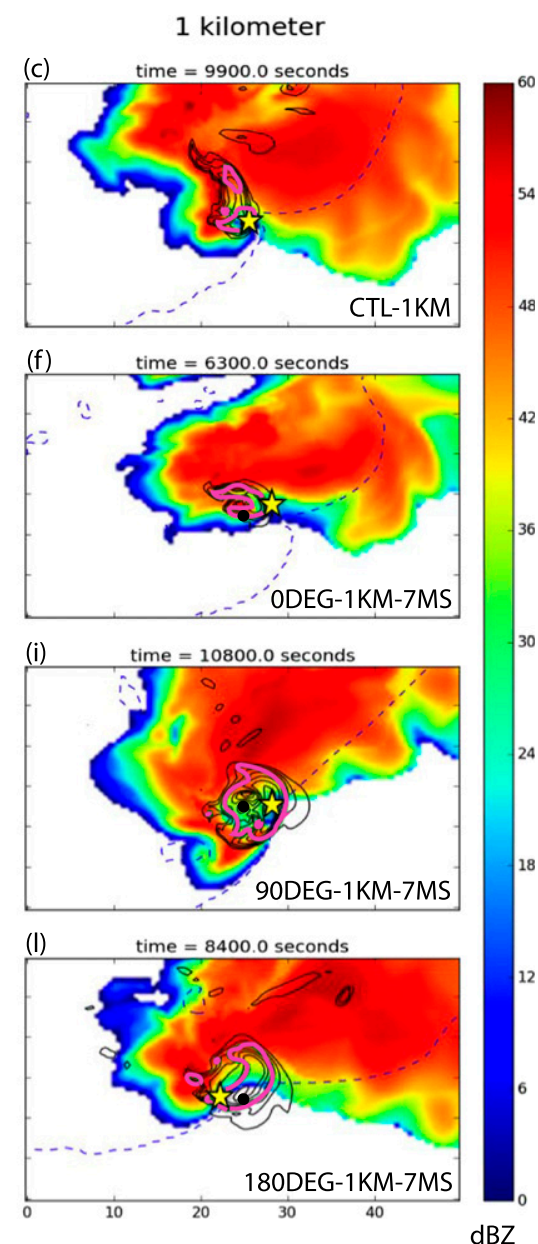

FIG. 6. As in Fig. 3, but for all $7 \mathrm{~m} \mathrm{~s}^{-1}$ shear magnitude simulations.

near the ground. Additionally, this alignment appears to be modulated, at least in part, by the low-level wind profile in the near-storm environment. The intensity of the mesocyclone aloft (i.e., the dynamic updraft forcing) does not appear to play a major part in the strengthening of near-surface vertical vorticity in this case, as UH is steady in three of the four simulations, particularly during the rapid intensification of vertical vorticity in the 0DEG-500M-7MS simulation. Indeed, even when UH increases substantially toward the end of the 90DEG500M-7MS simulation, the near-surface vertical vorticity does not increase as much as in the 0DEG-500M-7MS simulation.

\section{b. Influence of shear-layer depth}

Differences are also found among simulated supercells when the depth of the wind profile modification is varied, in addition to the orientation of the low-level shear vector. Figures 6-8 are similar to those discussed in the previous section but include results from two other sets of simulations where $d=250 \mathrm{~m}$ and $d=1 \mathrm{~km}$. In general, the patterns in overall storm structure seen in the 500-m simulations are reproduced at $d=250 \mathrm{~m}$ and $1 \mathrm{~km}$ as well, but differences are often amplified as the depth of the shear layer increases (Fig. 6). Though there are still notable differences in the reflectivity morphology among the $d=250$-m set of simulations (Figs. 6a,d,g,j), the overall storm structures are more similar in this set than in those with the deeper wind profile modifications. Like in the $d=500-\mathrm{m}$ simulations, the hook echo becomes progressively more "wrapped up," and the outflow boundary becomes more meridional and surges forward as the orientation angle transitions from $180^{\circ}$ (Fig. 6j) to $0^{\circ}$ (Fig. 6d); however, both patterns are more pronounced when $d=1 \mathrm{~km}$ (Figs. 6c,f,i,l). Interestingly, the size of the updraft tends to decrease as the depth of the wind profile modifications is increased, particularly for the control and $\alpha=0^{\circ}$ simulations. The 0DEG-1KM$7 \mathrm{MS}$ supercell is in decline; its small, relatively weak mesocyclone is entirely surrounded by outflow that is 

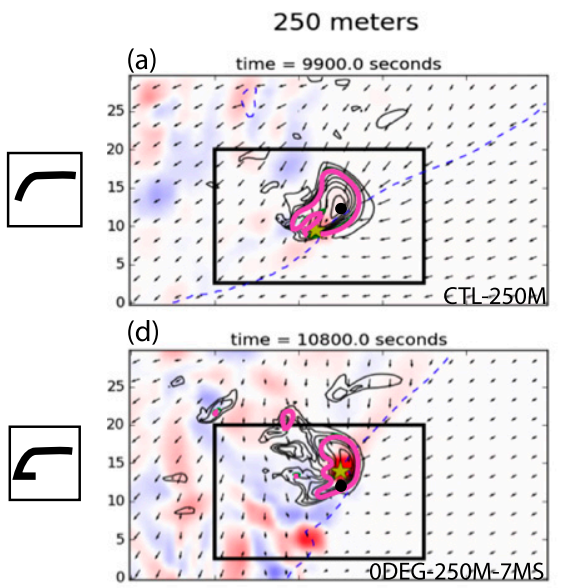

(g) time $=7500.0$ seconds
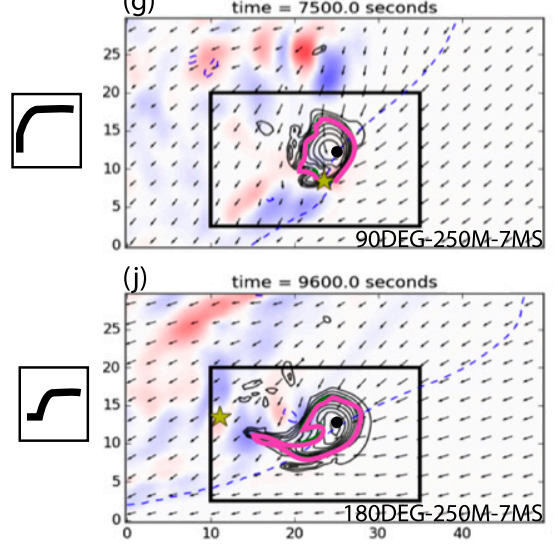

500 meters
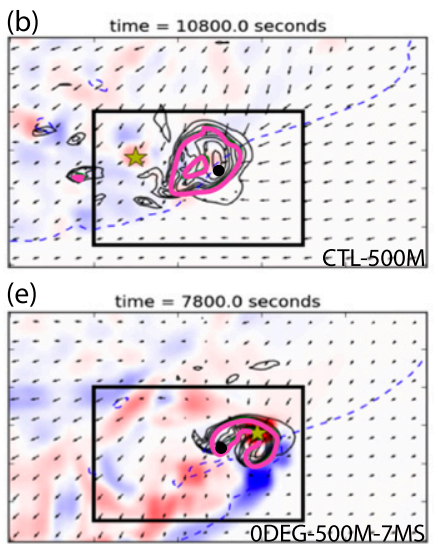

(h)
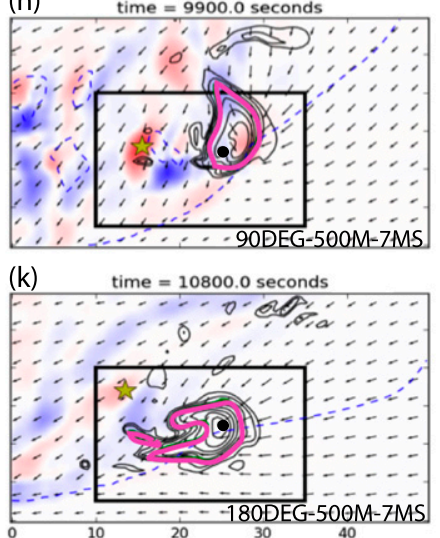

1 kilometer
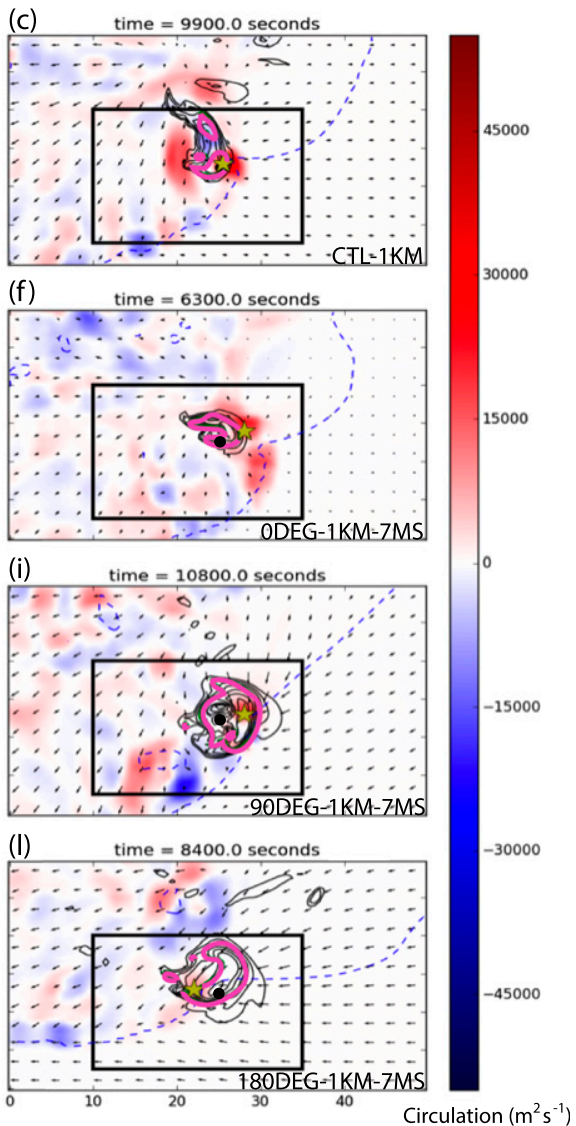

FIG. 7. As in Fig. 4, but for all $7 \mathrm{~m} \mathrm{~s}^{-1}$ shear magnitude simulations.

well occluded (Fig. 6f). Consequently, the reflectivity field is diminished in both size and intensity relative to the other simulations.

While morphological differences are more pronounced between the different $\alpha$ simulations for deeper values of $d$, near-surface circulation in the vicinity of the mesocyclone aloft appears to be more similar among the simulations with $d=1 \mathrm{~km}$ (Figs. 7c,f,i,l) than with $d=$ 250 (Figs. 7a,d,g,j) or 500 m (Figs. 7b,e,h,k). CTL-1KM (Fig. 7c) and 180DEG-1KM-7MS (Fig. 7l) have considerably larger values of circulation near and beneath the midlevel updraft than their smaller $d$ counterparts (cf. Figs. 7a,b,j,k). The clearly defined vorticity couplet structure seen in 0DEG-500M-7MS (Fig. 7e) is replaced by a broad area of weaker cyclonic circulation in the outflow-dominated 0DEG-1KM-7MS (Fig. 7f) simulation.

As seen in Fig. 8, the inverse relationship between mesocyclone separation distance and maximum nearsurface vertical vorticity is found regardless of the depth of the wind profile modifications. Unlike for shallower $d$, the separation distance is generally smaller and becomes slightly negative during the last hour of the CTL-1KM simulation (Fig. 8c). Correspondingly, the maximum near-surface vertical vorticity is larger at the end of this simulation than any other control simulation (cf. Figs. 8a,b). Mostly independent of $d$, the separation distance is generally smallest in the $\alpha=0^{\circ}$ simulations, and near-surface vertical vorticity is strongest (Figs. $8 \mathrm{~d}-\mathrm{f}$ ). The notable exception to this result is after $7000 \mathrm{~s}$ in the 0DEG-1KM-7MS simulation (Fig. 8f), when the separation distance becomes large and negative, indicating the circulation-rich outflow has surged well ahead of the overlying mesocyclone. As this occurs, near-surface vertical vorticity diminishes. Trends in separation distance and near-surface vertical vorticity are largely similar among all the $\alpha=90^{\circ}$ (Figs. $8 \mathrm{~g}-\mathrm{i}$ ) and $180^{\circ}$ (Figs. 8j-1) simulations except for around $7000 \mathrm{~s}$ in the 180DEG-1KM-7MS simulation (Fig. 81), where the separation distance becomes small for a time and nearsurface vertical vorticity increases.

Similar to the diminishing size of the mesocyclone aloft in all simulations as $d$ increases, $\mathrm{UH}$ often remains weaker as $d$ increases (green lines in Fig. 8), which is 

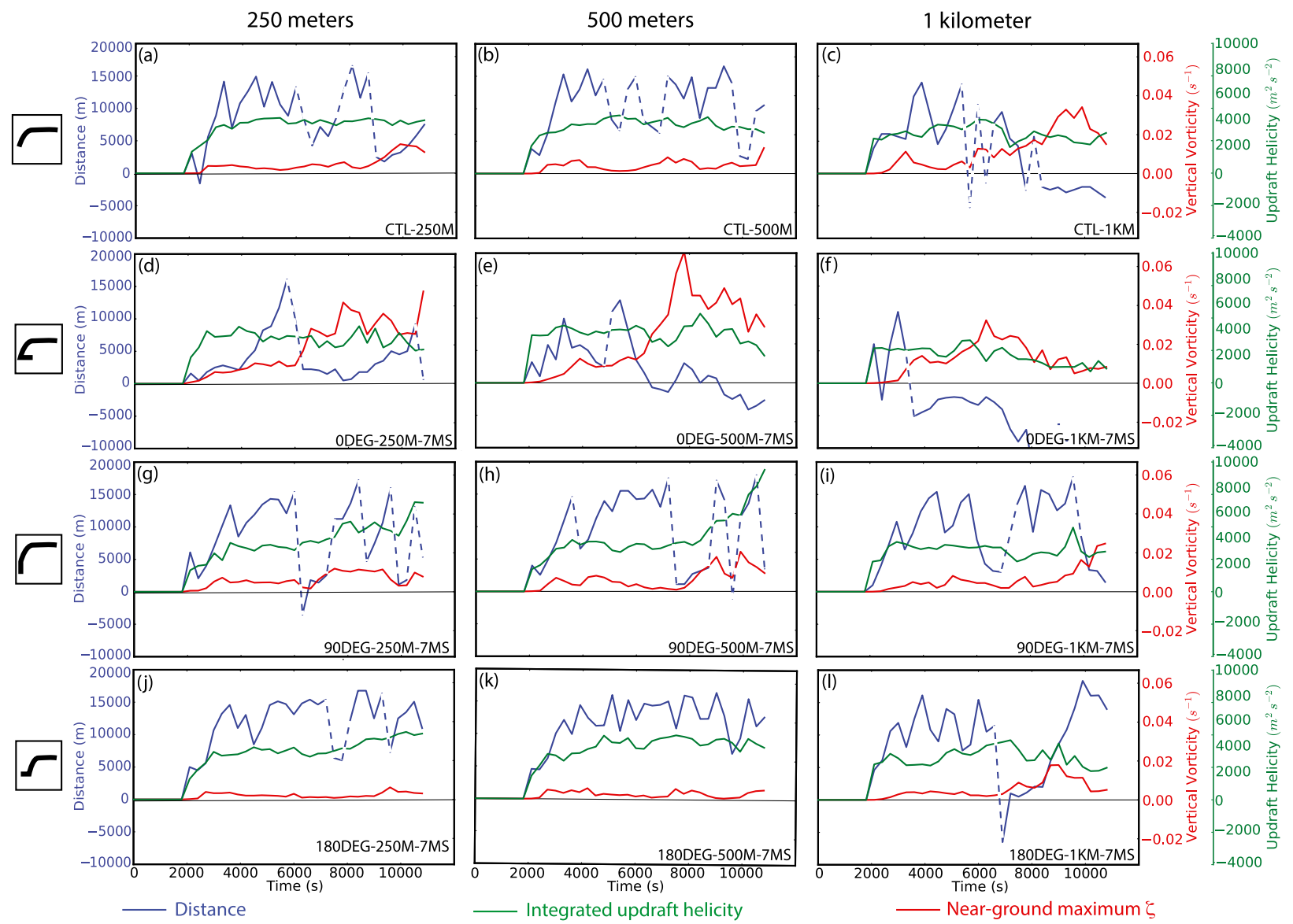

10000
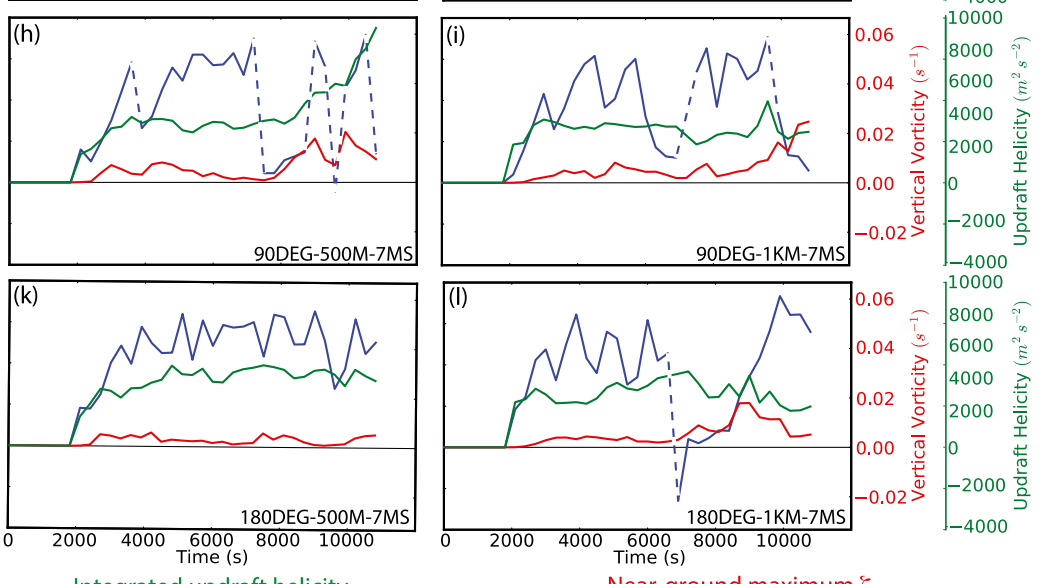

FIG. 8. As in Fig. 5, but for all $7 \mathrm{~m} \mathrm{~s}^{-1}$ shear magnitude simulations.

again likely due to decreases in SRH. Overall, the trends in maximum near-surface vertical vorticity in these simulations tend to follow trends in separation distance and do not appear to be tied to changes in UH. Indeed, at several times in different simulations [e.g., $10800 \mathrm{~s}$ in 90DEG-1KM-7MS (Fig. 8i), $10000 \mathrm{~s}$ in CTL-1KM (Fig. 8c), and after 6000 s in ODEG-250M-7MS (Fig. 8d)], near-surface vertical vorticity is at a maximum for the simulation despite no change in $\mathrm{UH}$. This reinforces the notion that differences in near-surface vertical vorticity in these simulations are largely related to the relative positioning of the updraft forcing with respect to the near-surface circulation rather than to changes in the maximum strength of the forcing.

\section{c. Influence of shear magnitude}

In a final set of simulations, the magnitude of the bulk shear was doubled to $15 \mathrm{~m} \mathrm{~s}^{-1}$ with $d=500 \mathrm{~m}$. As might be expected, morphological differences between the simulated storms with different $\alpha$ were amplified because of the greater shear and enhanced differences in low-level storm-relative winds (not shown). The
0DEG-500M-15MS simulation quickly became undercut by outflow, effectively terminating the storm's supercellular characteristics by the end of the simulation. The apparent relationship between mesocyclone separation distance and maximum near-ground vertical vorticity appears to apply to this set of simulations as well, though the separation for the $15 \mathrm{~m} \mathrm{~s}^{-1}$ cases generally remains relatively large (Fig. 9).

Notably, the maximum UH varies considerably among this set of experiments. Higher UH in the 90DEG-500M-15MS simulation is consistent with increased SRH in this case (Table 2). However, despite larger UH, near-surface vertical vorticity remains small, perhaps owing to the generally large mesocyclone separation distance (Fig. 9c). Indeed, the separation distance is often larger than that of the 90DEG-500MS7MS simulation, which is likely explained by increased northeasterly storm-relative winds restraining outflow in the 90DEG-500M-15MS simulation. In contrast, the 0DEG-500M-15MS has the weakest UH of all simulations (likely owing to a combination of weaker SRH in the near-storm environment and the early demise of the 


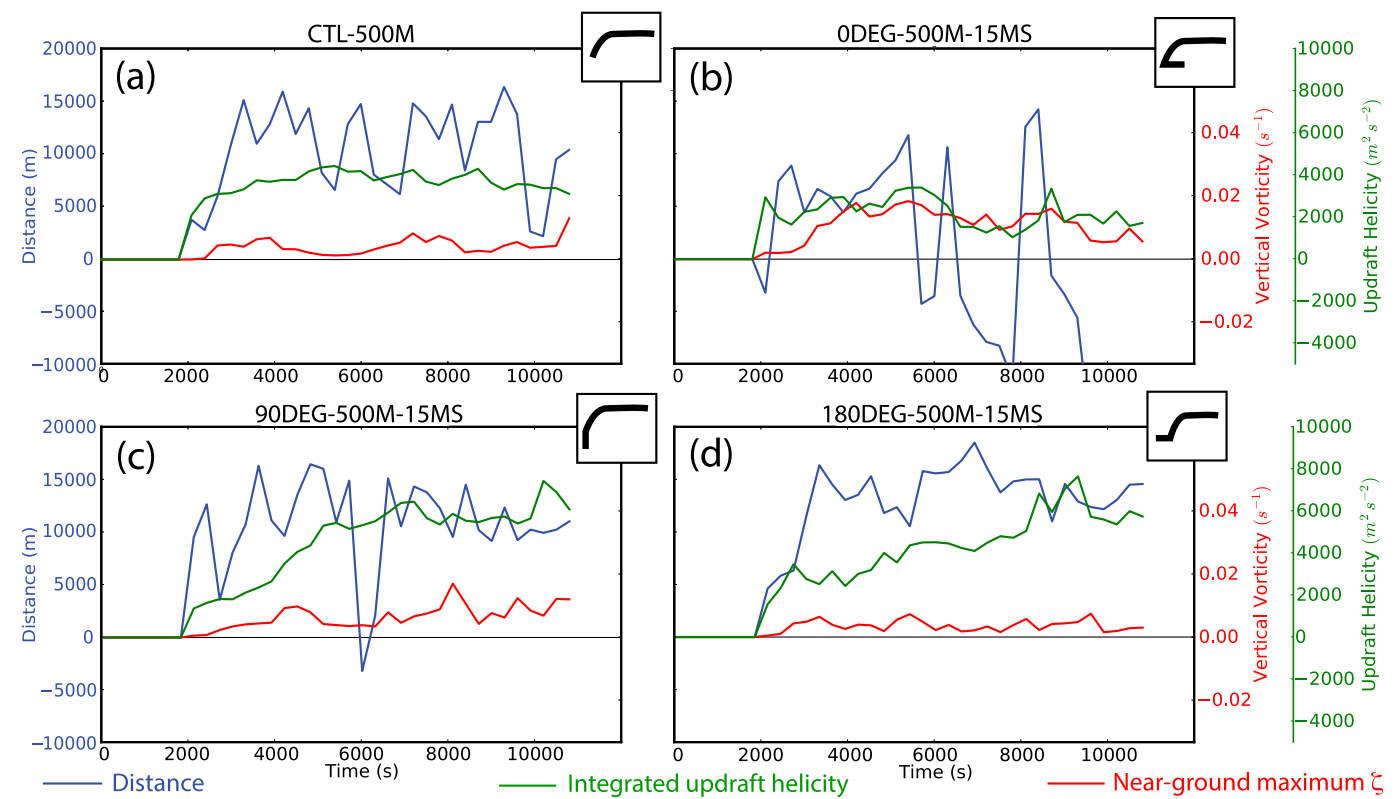

FIG. 9. As in Fig. 5, but for all $d=500-\mathrm{m}, 15 \mathrm{~m} \mathrm{~s}^{-1}$ shear magnitude simulations.

storm), yet it still has the largest near-surface vertical vorticity among the $15 \mathrm{~m} \mathrm{~s}^{-1}$ experiments. As before, the mesocyclone separation distance becomes negative as the outflow initially begins to undercut the mesocyclone aloft, and erratic separation distances (due to the lack of consistent near-surface and midlevel mesocyclones) characterize the dying storm over its last hour. The 180DEG-500M-15MS simulation has a consistently large separation distance coupled with weak nearsurface vertical vorticity (Fig. 9d) despite relatively large UH.

\section{d. Statistical analysis}

The simulation results presented above suggest that the orientation of the shear vector over relatively shallow layers can have a significant impact on the nearsurface vertical vorticity ultimately produced, possibly through the combined effects of the shear itself and changes in the storm-relative winds that accompany the modified low-level shear. These effects are seen for varying depths and magnitudes of the modifications to the low-level wind profile. The results also qualitatively suggest that one mechanism by which the wind profile modifications affect near-surface vertical vorticity is through the relative positioning of the near-surface circulation and midlevel mesocyclone. To explore this apparent relationship more quantitatively, we compare values of separation distance and near-surface vertical vorticity and circulation, as well as midlevel updraft helicity computed every $5 \mathrm{~min}$ over the last $2 \mathrm{~h}$ from each control and $7 \mathrm{~m} \mathrm{~s}^{-1}$ shear simulation (12 total simulations) across all shear orientation angles (Figs. 10 and 11$)^{2}$

In general, for the very narrow range of idealized atmospheric conditions simulated herein, the $\alpha=0^{\circ}$ simulations appear most likely to produce a tornadic vortex. ${ }^{3}$ Mesocyclone separation distance is smallest in the $\alpha=0^{\circ}$ supercells, with a median value close to zero (Fig. 10a). The largest values and smallest range of distances are found in the $\alpha=180^{\circ}$ simulations. Interestingly, despite a high median value of mesocyclone separation distance, the $\alpha=90^{\circ}$ supercells have a large range in the lower half of the distribution. This is consistent with some $\alpha=90^{\circ}$ supercells evolving to states with smaller distances and larger maximum near-surface vertical vorticity at more mature stages (Figs. 8g-i), perhaps indicating that at later times cold pools become more intense as the storm matures and are able to surge forward relative to the mesocyclone aloft despite adverse storm-relative winds opposing the gust front.

The inverse relationship between the magnitude of the separation distance and maximum near-surface vertical vorticity is evident when comparing the distribution of mesocyclone separation distance with that of

\footnotetext{
${ }^{2}$ The three $15 \mathrm{~m} \mathrm{~s}^{-1}$ simulations were not included owing to the premature demise of the 0DEG-500M-15MS supercell.

${ }^{3}$ The horizontal resolution of these simulations is too coarse to explicitly resolve tornadoes, so we rely on the assumption that larger maximum near-surface vertical vorticity indicates greater tornado likelihood.
} 

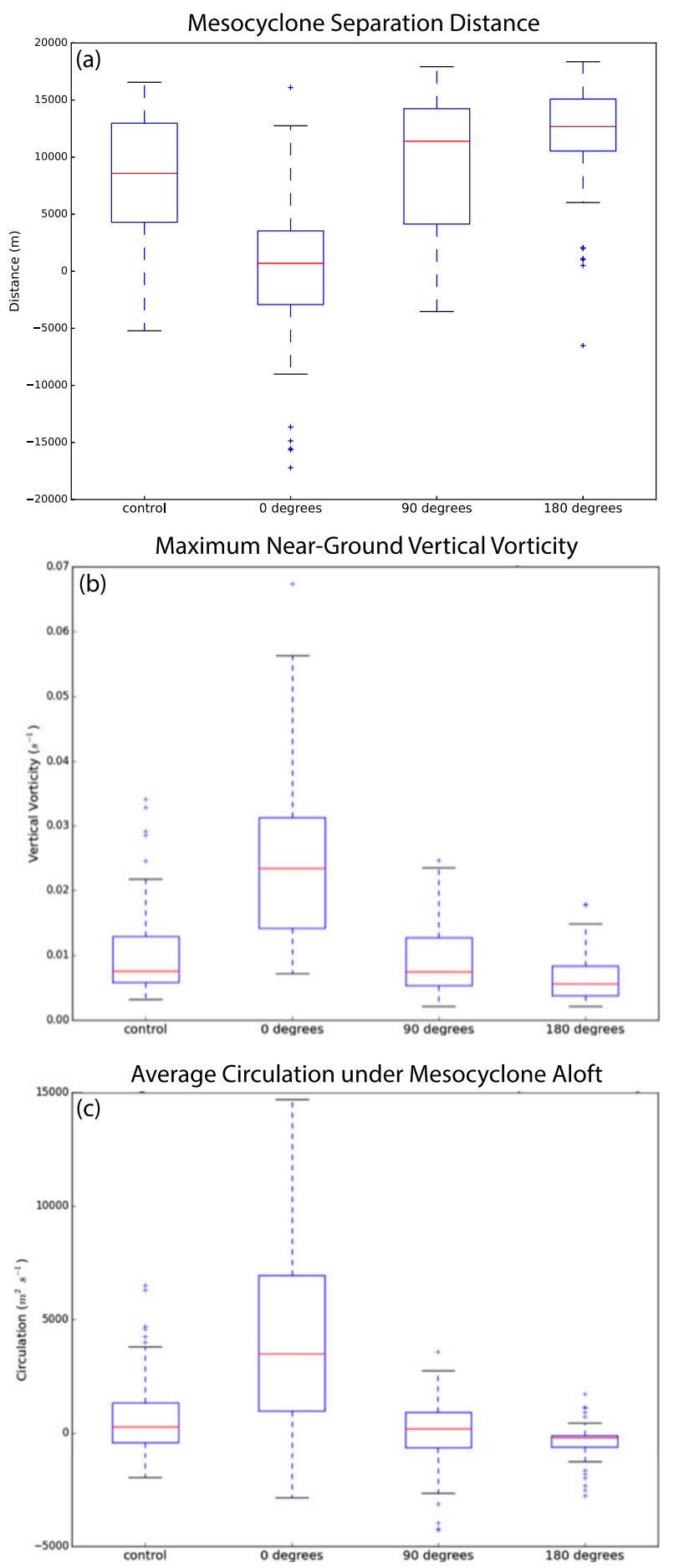

FIG. 10. Box-and-whisker plots showing distribution of (a) mesocylone separation distance, (b) maximum near-surface vertical vorticity, and (c) the average near-surface circulation below the area bounded by the $500 \mathrm{~m}^{2} \mathrm{~s}^{-2} \mathrm{UH}$ contour separated by shear orientation angle. Each distribution consists of samples taken at 5-min intervals over the last $2 \mathrm{~h}$ of each simulation for all $7 \mathrm{~m} \mathrm{~s}^{-1}$ bulk-shear simulations. In all plots, boxes represent the interquartile range, the whiskers extend 1.5 times the interquartile range in either direction, the median value is indicated by a red line, and outliers are marked with blue crosses. maximum near-surface vertical vorticity (Fig. 10b). Not surprisingly, the simulations with the smallest separation distance also tend toward greater average circulation beneath the mesocyclone aloft (Fig. 10c). Figure 11 shows time series of both the average near-surface circulation and the fraction of grid points with appreciable $\left(>5000 \mathrm{~m}^{2} \mathrm{~s}^{-1}\right)$ near-surface circulation beneath the low-level mesocyclone. These provide a different perspective than the mesocyclone separation distance by quantifying the relative overlap of the low-level mesocyclone and areas where near-surface circulation is generated rather than simply the distance between maximum values at different heights. Moreover, focusing on the low-level mesocyclone (i.e., the base of the vertical layer used in the UH integration for mesocyclone separation distance) for this analysis will reveal any biases in mesocyclone separation distance caused by mesocyclone tilt well above $1 \mathrm{~km}$. The simulations with the greatest average near-surface circulation (Fig. 11a) and fraction of grid points with appreciable circulation (Fig. 11b) directly beneath the low-level mesocyclone (0DEG-500M-7MS, 0DEG-250M-7MS, CTL-1KM, and even 180DEG-1KM-7MS) tend to have the largest overall values of near-ground vertical vorticity and periods of small mesocyclone separation distance (cf. Figs. 8c-e,1). Furthermore, despite brief periods of small mesocyclone separation distance in other simulations (90DEG-500M-7MS and 90DEG-250M-7MS), there seems to be insufficient overlap in the near-surface circulation and low-level mesocyclone for very strong nearground vertical vorticity to form. Thus, regardless of the metric of alignment used in the analysis, in the $\alpha=0^{\circ}$ simulations, there appears to be a much greater frequency of prerequisite conditions for near-surface vortex stretching (i.e., surface circulation located below a mesocyclone), while this condition is rarely met for the $\alpha=180^{\circ}$ wind profiles. This notion is reinforced by the relatively strong negative correlation between mesocyclone separation distance and maximum nearsurface vertical vorticity in all of the simulations (Fig. 12a). Correlations are performed with a 10-min lag on the maximum near-surface vertical vorticity from the abscissa variables in Fig. 12 on the assumption that vertical vorticity is the dependent variable and requires some time before influences of the independent variable are manifest. Indeed, with the time lag, correlation coefficients are generally higher.

While there is considerable evidence that the intensity of near-surface vertical vorticity in our experiments is closely tied to the alignment between the midlevel mesocyclone and regions of circulation-rich air at the ground, it is possible that the observed relationship may be due to other physical processes. For instance, one 

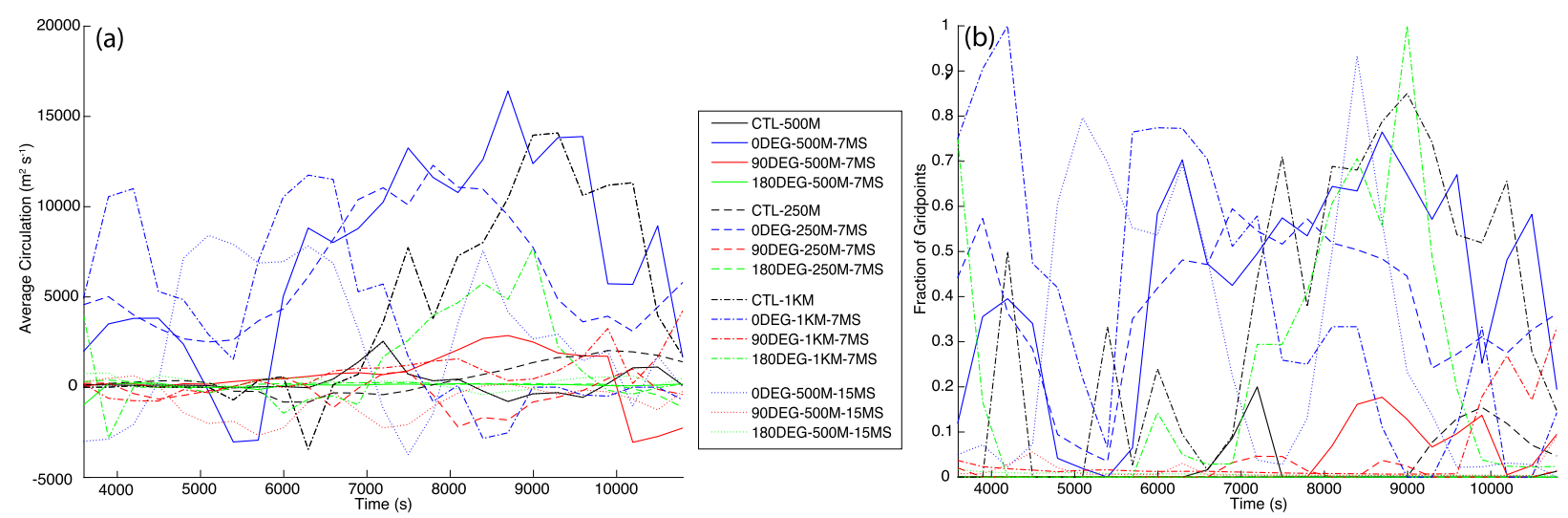

FIG. 11. Time series of (a) the average near-surface circulation and (b) fraction of grid points with near-surface circulation exceeding $5000 \mathrm{~m}^{2} \mathrm{~s}^{-1}$ beneath the low-level mesocyclone, defined as the area at $z=1 \mathrm{~km}$ where vertical velocity is greater than $1 \mathrm{~ms}$ and circulation exceeds $10000 \mathrm{~m}^{2} \mathrm{~s}^{-1}$.

alternative hypothesis may be that the low-level wind profile alterations modify the intensity of the mesocyclone aloft and that these intensity differences are the root cause of variations in near-surface vertical vorticity. If this were the case, however, one would expect 1-6-km $\mathrm{UH}$ and maximum near-surface vertical vorticity to be strongly positively correlated. This is not the case (Fig. 12b). Only the control simulations display a relatively strong correlation between UH and maximum near-surface vertical vorticity, but it is a negative correlation. Indeed, the correlation for the total sample is weakly negative. The strongest positive correlation is in the $\alpha=0^{\circ}$ case. Though still fairly weak, this suggests that it is only in the cases where mesocyclone separation distance is small that the intensity of the mesocyclone aloft can modulate near-surface vertical vorticity intensity.

\section{e. Analysis of dynamic low-level updraft forcing}

Our hypothesis for the relationship between separation distance and near-surface vertical vorticity hinges on the idea that a supercell mesocyclone is associated with an upward-directed dynamic vertical perturbation pressure gradient force (VPPGF) at low levels (below the vertical vorticity maximum in the mesocyclone). Thus, when the mesocyclone aloft is above positive circulation near the surface, vertical vorticity may intensify via lifting and stretching of the negatively buoyant outflow air in a dynamically driven updraft. If we denote our hydrostatically balanced base state by $\overline{\theta_{\rho}}$ and $\bar{\pi}$, where $\theta_{\rho}$ is the density potential temperature and $\pi \equiv\left(p / p_{0}\right)^{R / c_{p}}$ is the Exner function, then the vertical accelerations in the flow are described by

$$
\frac{D w}{D t}=-c_{p} \overline{\theta_{\rho}} \frac{\partial \pi^{\prime}}{\partial z}+B
$$

where $w$ is the vertical component of velocity, $\pi^{\prime}=\pi-\bar{\pi}$ is the perturbation Exner function, which plays the role of perturbation pressure, $c_{p}$ is the specific heat capacity at constant pressure, and $B=g\left(\theta_{\rho}-\overline{\theta_{\rho}}\right) / \overline{\theta_{\rho}}=$ $g \theta_{\rho}^{\prime} \sqrt{\theta_{\rho}}$ is the acceleration due to buoyancy. The terms on the right-hand side of (1) represent vertical accelerations due the vertical pressure gradient and the buoyancy, respectively. Near the ground in a supercell cold pool, where we are most concerned with vorticity stretching in this study, the contributions from the buoyancy term are largely negative. Thus, vertical gradients of nonhydrostatic pressure perturbations $\partial \pi^{\prime} / \partial z$ are assumed to be the dominant driving force of low-level updrafts within supercell outflow.

To calculate the VPPGF, perturbation pressures were decomposed following the method of Hastings and Richardson (2016; for a full description, see their appendix A), which is similar to the method employed by Rotunno and Klemp (1982, 1985), wherein the full nondimensional pressure perturbation $\pi^{\prime}$ is separated into buoyant $\left(\pi_{B}^{\prime}\right)$, nonlinear dynamic $\left(\pi_{\mathrm{ND}}^{\prime}\right)$, and linear dynamic $\left(\pi_{\mathrm{LD}}^{\prime}\right)$ components:

$$
\pi^{\prime}=\pi_{B}^{\prime}+\pi_{\mathrm{ND}}^{\prime}+\pi_{\mathrm{LD}}^{\prime},
$$

where $\pi_{B}^{\prime}$ and $\pi_{\mathrm{LD}}^{\prime}$ are calculated explicitly using a Poisson solver with Neumann lateral boundary conditions and $\pi_{\mathrm{ND}}^{\prime}$ is found as the residual (see Hastings and Richardson 2016). The $\pi_{B}^{\prime}$ term tends to be negative (positive) above (below) the local buoyancy minimum, $\pi_{\mathrm{ND}}^{\prime}$ is positive in regions of deformation and negative in regions of vorticity, and $\pi_{\mathrm{LD}}^{\prime}$ is positive (negative) upshear (downshear) of updrafts and negative (positive) upshear (downshear) of downdrafts. The total vertical perturbation pressure gradient is thus described as 

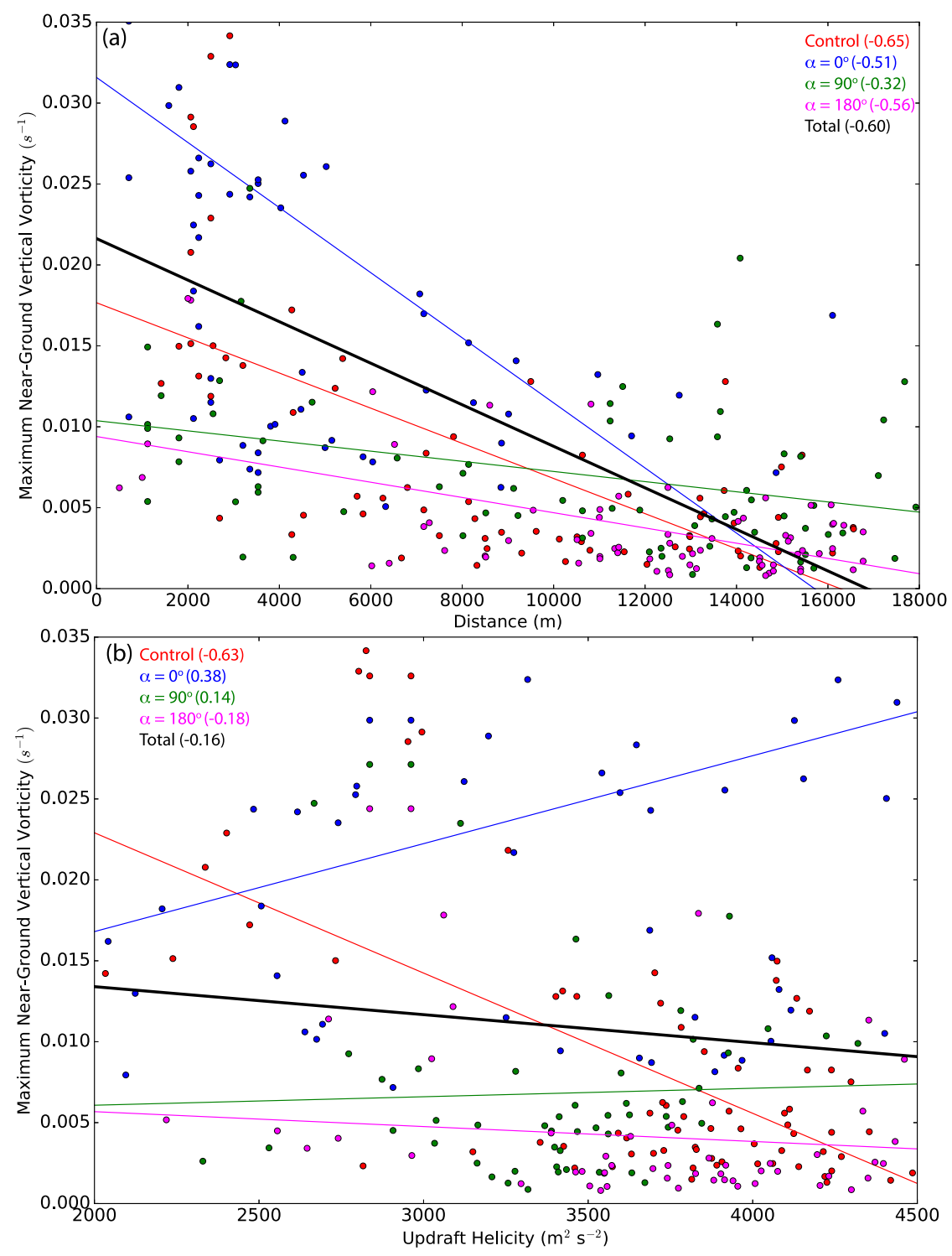

FIG. 12. Scatterplots of maximum near-surface vertical vorticity taken at 5-min intervals over the last $2 \mathrm{~h}$ of each $7 \mathrm{~m} \mathrm{~s}^{-1}$ bulk-shear simulation as a function of (a) mesocyclone separation distance and (b) maximum 1-6-km UH. The ordinate is lagged by $10 \mathrm{~min}$ from the abscissa. The linear regression lines and correlation coefficients are shown for the control (red), $\alpha=0^{\circ}$ (blue), $\alpha=90^{\circ}$ (green), $\alpha=180^{\circ}$ (magenta), and all (black) simulations.

$$
\frac{\partial \pi^{\prime}}{\partial z}=\frac{\partial \pi_{B}^{\prime}}{\partial z}+\frac{\partial \pi_{\mathrm{ND}}^{\prime}}{\partial z}+\frac{\partial \pi_{\mathrm{LD}}^{\prime}}{\partial z} .
$$

In the negatively buoyant outflow, the buoyant part of the VPPGF tends to be everywhere positive [and countered by the negative buoyancy acceleration in (1)] and thus does not contribute specifically to driving the updraft. Therefore, we focus on only the dynamic VPPGF, which is defined as $-c_{p} \theta_{\rho}\left(\partial \pi_{\mathrm{ND}}^{\prime} / \partial z+\partial \pi_{\mathrm{LD}}^{\prime} / \partial z\right)$. In general, the total dynamic VPPGF tends to be dominated by the nonlinear effects near the surface in these simulations (not shown).
Figure 13 shows the juxtaposition of near-surface circulation with the dynamic VPPGF at $500 \mathrm{~m}$ above the surface for each of the cases with $d=500 \mathrm{~m}$. In general, the mesocyclone aloft (as outlined by the green $\mathrm{UH}$ contour) is associated with positive dynamic VPPGF in all four panels. The largest dynamic forcing in Fig. 13 occurs in the 90DEG-500M-7MS simulation (Fig. 13c), consistent with the larger SRH in this case. At the time of the figure (7200 s), the maximum VPPGF in the 90DEG-500M-7MS case is roughly aligned with a region of weak vertical circulation at the surface, which 

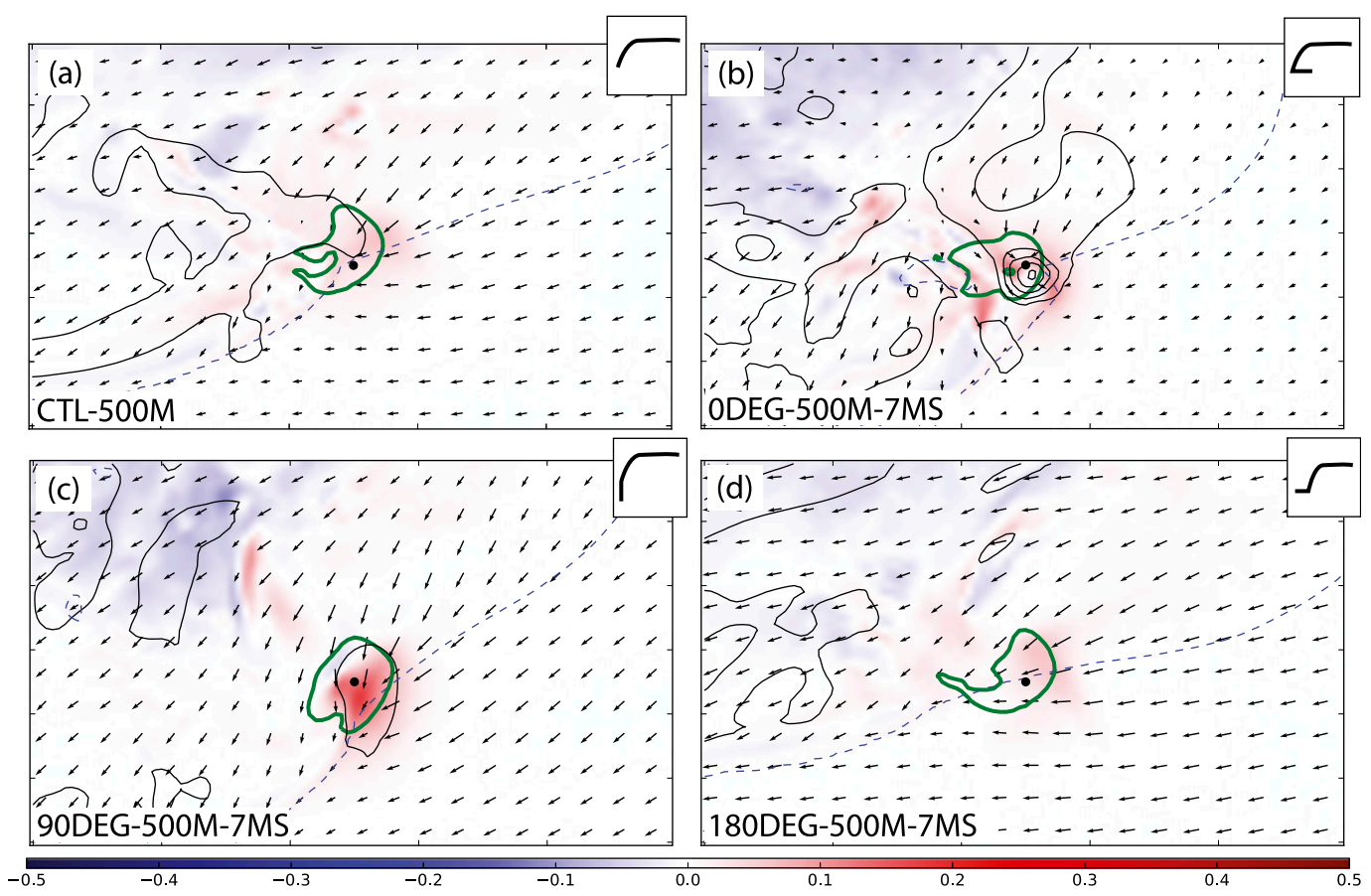

Dynamic VPPGF $\left(\mathrm{m} \mathrm{s}^{-2}\right)$

FIG. 13. Plan views of dynamic VPPGF $500 \mathrm{~m}$ above the lower model surface (shaded), circulation at the lower model surface (black contours of $0.5 \times 10^{3}, 1 \times 10^{4}, 2 \times 10^{4}, 3 \times 10^{4}, 4 \times 10^{4}$, and $5 \times 10^{4} \mathrm{~m}^{2} \mathrm{~s}^{-1}$ ), storm-relative surface winds, and the $-1-\mathrm{K}$ surface potential temperature contour (dashed blue) for the (a) CTL-500M, (b) 0DEG-500M-7MS, (c) 90DEG-500M-7MS, and (d) 180DEG-500M-7MS simulation at $t=7200 \mathrm{~s}$. The solid green contour, representing the outline of the mesocyclone aloft, is the $600 \mathrm{~m}^{2} \mathrm{~s}^{-2} \mathrm{UH}$ outline, and the black dot is the maximum integrated $\mathrm{UH}$.

leads to a period of relatively rapid near-surface vorticity growth over the following $\sim 20 \mathrm{~min}$ (cf. Fig. $5 \mathrm{c}$ ). However, around $t=9000 \mathrm{~s}$, the maximum near-ground vertical vorticity becomes steady following a period of increased separation distance. By contrast, the dynamic VPPGF in the 0DEG-500M-7MS simulation is relatively weak and diffuse (Fig. 13b). Despite this apparent weakness, the dynamic forcing in this case is aligned with a region of robust circulation at the surface, resulting in a period of rapid and sustained near-surface vorticity intensification (Fig. 5b). Note that this vorticity amplification follows a period of steadily decreasing separation distance, which is suggestive of a gradual improvement in the alignment of the near-ground and midlevel mesocyclones leading up to the time in Fig. 13b. In the remaining two simulations (CTL-500M and 180DEG-500M-7MS), the mesocyclone and associated low-level dynamic forcing remain largely ahead of zones of surface circulation in the cold pool (Figs. 13a,d), while the vorticity at the surface remains small for the duration of the simulations (Figs. 5a,d).

The foregoing analysis suggests that potentially significant differences in both the strength of near-surface circulation and low-level dynamic updraft forcing can exist between simulations as a result of changes in the orientation of the low-level wind profile. It seems, however, that more important than the maximum strength of the dynamic forcing is the collocation of the best dynamic forcing with the low-level circulation maximum. For example, the 0DEG-500M-7MS simulation has a notably weaker dynamic VPPGF than the 90DEG500M-7MS simulation, which is ostensibly detrimental to near-surface vortex intensification; however, the 0DEG-500M-7MS has a greater availability of near-surface circulation, particularly at earlier simulation times. Furthermore, the smaller mesocyclone separation distance over a more sustained period of time in the 0DEG-500M7MS simulation (Fig. 5) suggests that the near-surface circulation is better aligned with the low-level dynamic forcing over a longer timer interval than in the $\alpha=90^{\circ}$ simulation. Both of these facts lead to larger maximum near-surface vertical vorticity in the 0DEG-500M-7MS simulation than is possible in the 90DEG-500M-7MS simulation.

Apart from the collocation of near-surface circulation with an upward-directed VPPGF, outflow air must not be too negatively buoyant (e.g., Markowski et al. 2002; Grzych et al. 2007), or else the buoyancy term in (1) will be strongly negative and dominate the upward VPPGF, 

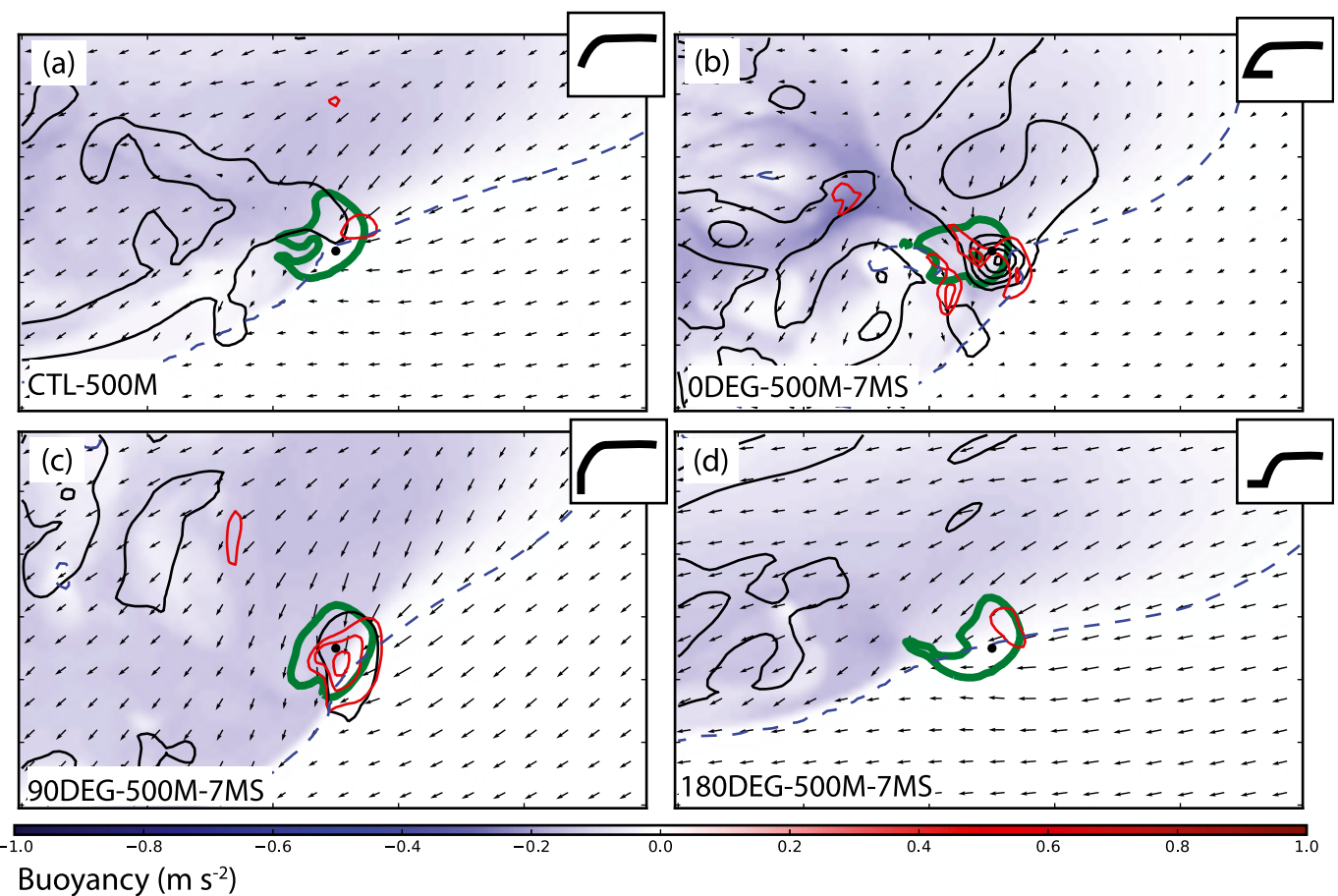

FIG. 14. Plan views of buoyancy at the lowest grid level (shaded), circulation at the lower model surface (black contours of $0.5 \times 10^{3}, 1 \times 10^{4}, 2 \times 10^{4}, 3 \times 10^{4}, 4 \times 10^{4}$, and $5 \times 10^{4} \mathrm{~m}^{2} \mathrm{~s}^{-1}$ ), storm-relative surface winds, the -1 -K surface potential temperature contour (dashed blue), and dynamic VPPGF $500 \mathrm{~m}$ above the lower model surface (red contours every $0.05 \mathrm{~m} \mathrm{~s}^{-2}$ ) for the (a) CTL-500M, (b) 0DEG-500M-7MS, (c) 90DEG-500M-7MS, and (d) $180 \mathrm{DEG}-500 \mathrm{M}-7 \mathrm{MS}$ simulations at $t=7200 \mathrm{~s}$. The solid green contour, representing the outline of the mesocyclone aloft, is the $600 \mathrm{~m}^{2} \mathrm{~s}^{-2} \mathrm{UH}$ outline, and the black dot is the maximum integrated UH.

preventing strong low-level updrafts and vorticity stretching. We compare the outflow buoyancy between the four varying $\alpha$ simulations where $d=500 \mathrm{~m}$ and the low-level bulk shear is $7 \mathrm{~m} \mathrm{~s}^{-1}$ in Fig. 14. In the region of each storm where there is overlap between the upward dynamic VPPGF and at least modest positive circulation at the surface, outflow buoyancy is similar among three of the four simulations. The exception is the 180DEG500M-7MS simulation, where outflow buoyancy is less negative, but no such region of overlap exists owing to the large mesocyclone separation distance. Consequently, it appears that changes in outflow buoyancy may be eliminated as an explanation of the variations in vortex intensification seen in these simulations. Thus, at least in our experiments, it seems that the greatest influence of the low-level wind profile changes is through their effects on the separation distance between the near-surface circulation maximum and the favorable dynamic forcing provided by the mesocyclone aloft.

\section{f. Unexpected intensity of near-ground vertical vorticity in the $\alpha=0^{\circ}$ simulations}

Perhaps the most striking result from these simulations is that the $\alpha=0^{\circ}$ simulations tend to have the strongest near-surface vertical vorticity, when previous studies on this topic suggest that this hodograph shape is not conducive to tornadoes (e.g., Wicker 1996; Miller 2006; Esterheld and Guiliano 2008; Kis and Straka 2010; Nowotarski and Jensen 2013; Parker 2014; Coffer and Parker 2017). Several studies have suggested that the predominately crosswise low-level horizontal vorticity, weaker storm-relative winds opposing the gust front, and easterly shear associated with the $\alpha=0^{\circ}$ hodographs should result in outflow-dominant storms wherein the lowlevel circulation surges ahead of the storm (e.g., Frame and Markowski 2013; Nowotarski and Jensen 2013; Parker 2014). Some similarity exists in the near-ground circulation field of the 0DEG-500M-7MS simulation (Fig. 4b) and the pattern seen in the Coffer and Parker (2017) nontornadic simulation (cf. their Figs. 3h and 18b). Both simulations exhibit a couplet of positive and negative circulation oriented along a meridional axis; however, in our simulations, these features are sustained longer and located below the mesocyclone aloft.

A comparison of cold pool and updraft position for the CTL-500M and 0DEG-500M-7MS simulations is shown in Figs. 15 and 16. Even as early as 3600 s, outflow and weak vertical vorticity are located beneath the 

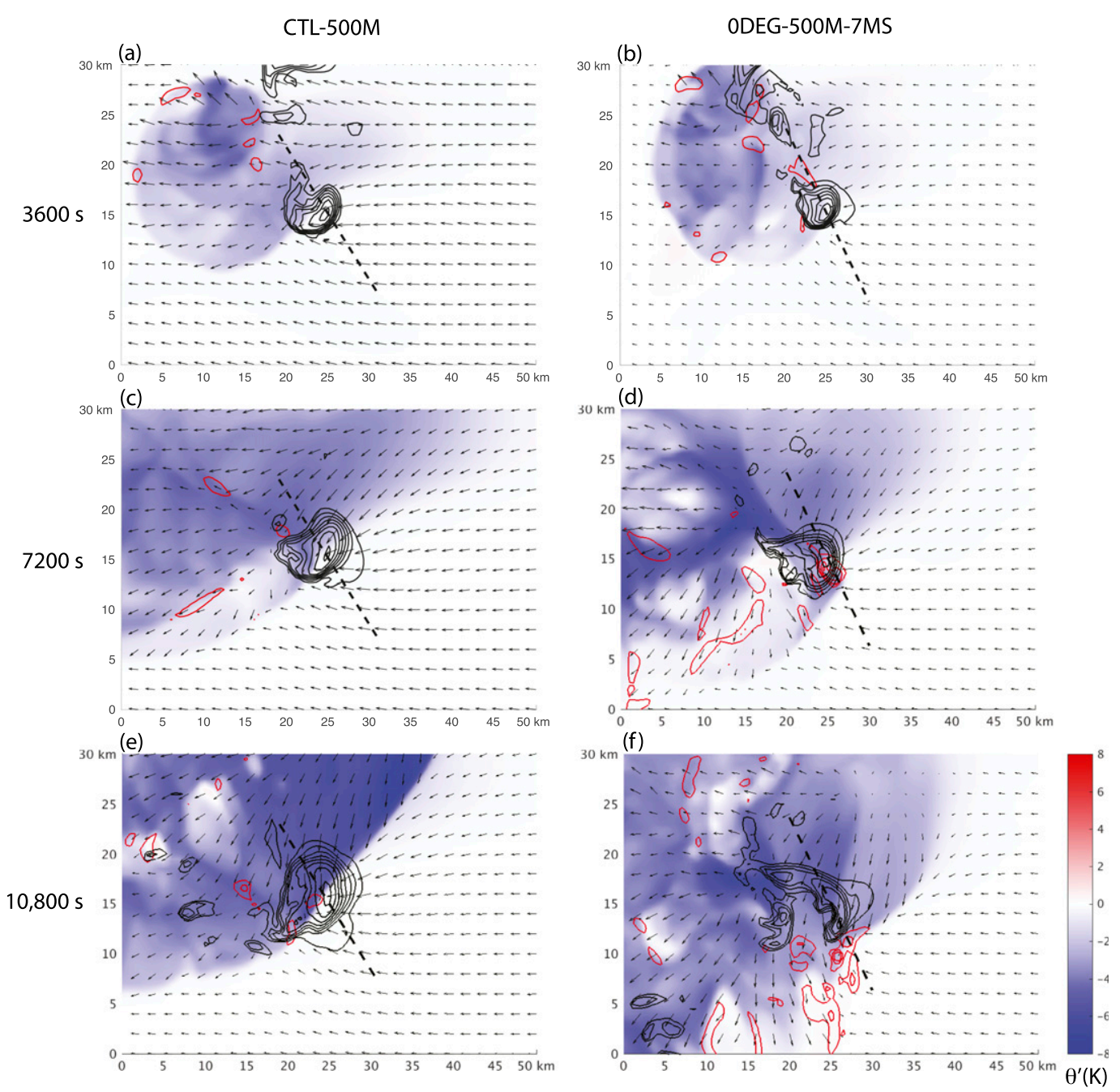

FIG. 15. Plan views, centered on the 1-6-km integrated UH maximum, of potential temperature perturbation at the lowest grid level (shaded), vertical velocity at $z=4 \mathrm{~km}$ (black contours at $5 \mathrm{~m} \mathrm{~s}^{-1}$ intervals), vertical vorticity at the lowest grid level (red contours at $2.5 \times 10^{-3}$ and $5 \times 10^{-3} \mathrm{~s}^{-3}$ and $1 \times 10^{-2}-\mathrm{s}^{-1}$ increments thereafter), and storm-relative surface winds for the (a),(c),(e) CTL-500M and (b),(d),(f) 0DEG-500M-7MS simulations at hourly intervals. The black dashed line indicates the footprint of the vertical cross sections shown in Fig. 16.

midlevel storm updraft in the 0DEG-500M-7MS simulation (Figs. 15b and 16b), whereas the leading edge of the outflow is located well northwest of the updraft core in the CTL-500M simulation at this time (Figs. 15a and 16a). Given similar cold pool depths and intensity, this difference in outflow position is likely explained, at least in part, by the low-level wind profile in the plane perpendicular to the gust fronts. It is apparent from Fig. 16 that there are both weaker storm-relative winds opposing the gust front and shear oriented toward the gust front over its depth in the 0DEG-500M-7MS simulation compared with the CTL-500M simulation, which idealized density current simulations suggest should promote greater forward motion (e.g., Xu 1992; Xue 2000). By $7200 \mathrm{~s}$, the outflow has become colder, and the gust front has surged forward relative to the updraft in both simulations (Figs. 15c,d and 16c,d), though near the surface, the CTL-500M gust front still trails the 0DEG-500M7MS simulation. At this time, it is clear that the 0DEG500M-7MS simulation, despite (or perhaps because of) more forward-propagating outflow, has a smaller mesocyclone separation distance and develops stronger near-surface vertical vorticity (cf. Fig. 5). By the end of the simulations, the leading edge of the outflow and near-surface vertical vorticity maximum has surged well forward of the core of the updraft in the 0DEG-500M$7 \mathrm{MS}$ simulation (Figs. $15 \mathrm{f}$ and $16 \mathrm{f}$ ). Though it might be expected that near-ground vertical vorticity should quickly decrease in this scenario, only a slow decline in the strength of near-surface vertical vorticity (Fig. 5b) 

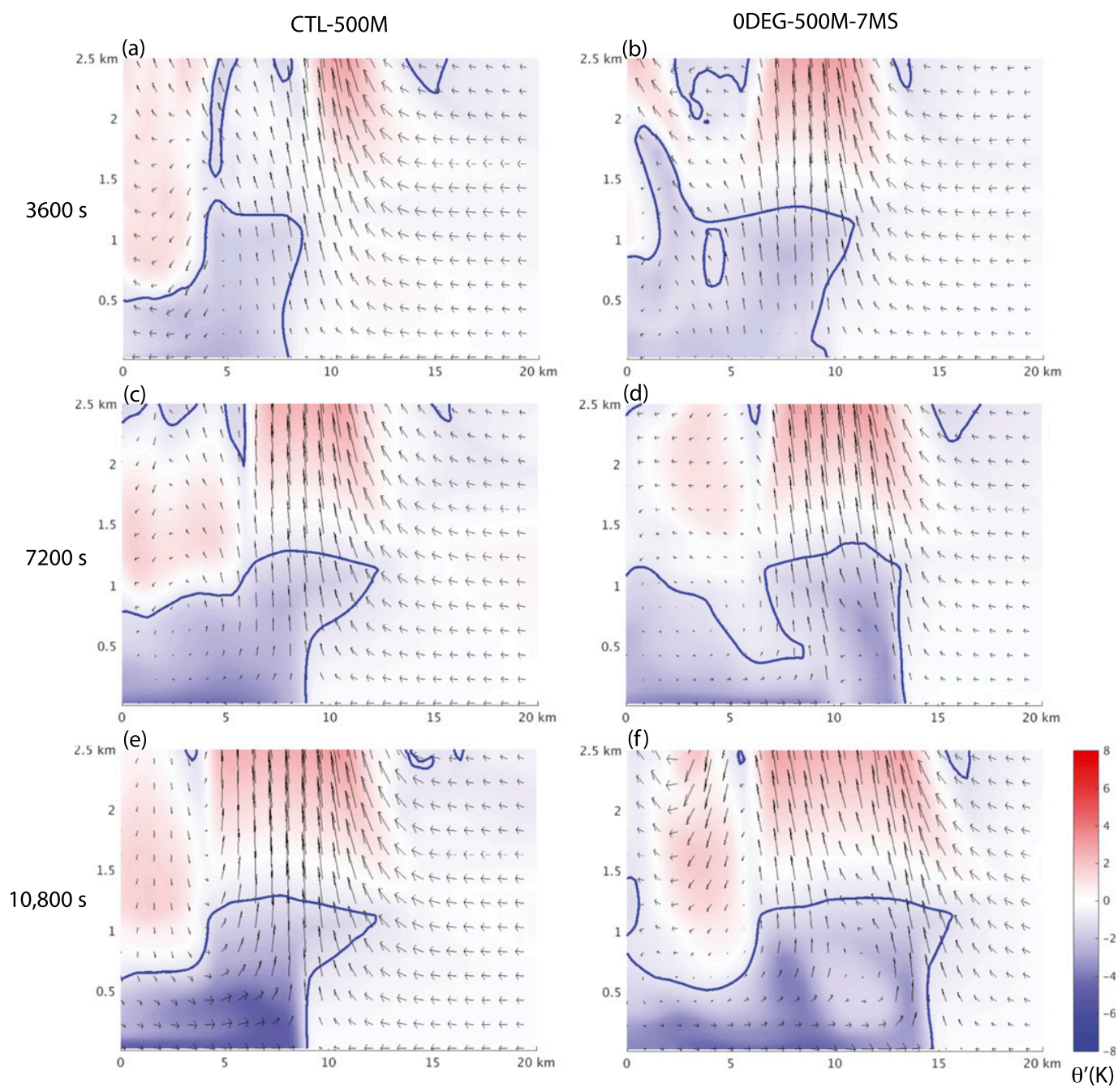

FIG. 16. Vertical cross sections, centered on the 1-6-km integrated UH maximum, along the planes perpendicular to the average gust-front orientation for each simulation (defined in section 2 and reported in Table 3) identified in Fig. 15 of potential temperature perturbation (shaded) and storm-relative velocity in the plane of the cross section for the (a),(c),(e) CTL-500M and (b),(d),(f) 0DEG-500M-7MS simulations at hourly intervals. The $-1-\mathrm{K}$ potential temperature perturbation contour is shown in blue.

occurs. This is consistent with observations from Dowell and Bluestein (2002), where tornadoes were found to persist well after they became displaced from the midlevel updraft by as much as several kilometers. At this time, the leading edge of the outflow has reached its most forward position relative to the updraft, and appreciable vertical vorticity finally begins to develop beneath the updraft in the CTL-500M simulation (Figs. 15e and 16e).

Thus, in our simulations, outflow does indeed tend to surge forward in the 0DEG-500M-7MS simulation, as expected; however, this actually promotes low-level rotation because it allows for smaller mesocyclone separation distances at earlier times in our simulations. It is only when the depth and intensity of the low-level shear are increased that this shear orientation results in a weakened storm and weaker near-surface vertical vorticity but even then only at later times. Because the motion of the outflow is due to a combination of factors, one of which is the negative buoyancy of the outflow, it is likely that storms with colder cold pools than those simulated here may result in more favorable (unfavorable) mesocyclone separation distances for other (the $\alpha=0^{\circ}$ ) hodograph shapes. Thus, for different thermodynamic environments or deep-layer wind profiles that promote different hydrometeor distributions and/or changes in latent cooling of hydrometeors, different shear orientations may be most favorable for tornadoes. 


\section{Summary and conclusions}

A series of idealized numerical simulations of supercell thunderstorms with varying environmental lowlevel wind profiles was performed. In each suite of experiments, the orientation of the low-level wind shear vector is varied and compared with a control simulation with no vertical wind shear over the prescribed layer. The experiments were repeated for three different depths of the imposed shear layers $(250 \mathrm{~m}, 500 \mathrm{~m}$, and $1 \mathrm{~km}$ ), as well as for two different magnitudes of the bulk shear ( 7 and $\left.15 \mathrm{~m} \mathrm{~s}^{-1}\right)$. These experiments were designed to test the hypothesis that variations in low-level shear and/or storm-relative winds can modulate the intensification of near-surface vertical vorticity through their effects on the relative positioning of the dynamic updraft forcing associated with the mesocyclone aloft and the regions of sustained near-surface circulation. The results of these experiments support the following conclusions:

(i) Modifications to the low-level wind profile, even over quite shallow layers, can have a large influence on overall supercell morphology, including precipitation distribution, updraft size and shape, and gust-front orientation.

(ii) Variations in the orientation of the low-level shear vector and corresponding changes in the low-level storm-relative winds can also modulate the magnitude of near-surface vertical vorticity.

(iii) The horizontal distance between the near-surface circulation maximum and 1-6-km updraft helicity maximum, or the "mesocyclone separation distance," varies as a function of the orientation of the low-level wind profile. This is primarily because the supercell outflow and embedded circulation maxima are either restrained or surge forward relative to the mesocyclone aloft, depending on the orientation of the low-level winds.

(iv) Maximum near-surface vertical vorticity is negatively correlated with mesocyclone separation distance for all hodographs in this study. The strongest (most negative) correlation was found when the vertical vorticity was lagged by $10 \mathrm{~min}$, suggesting that the decreasing separation distance tends to lead the vorticity increase in time.

(v) The effects on mesocyclone separation distance and the intensity of near-surface vertical vorticity as a function of shear orientation persist for varying depths (as shallow as $250 \mathrm{~m}$ ) and bulk-shear magnitudes.

(vi) The most rapid amplification and highest values of near-surface vertical vorticity result when the favorable dynamic vertical perturbation pressure gradient force associated with the mesocyclone aloft is collocated with the near-surface circulation maximum.

(vii) As a condition for vorticity amplification, the degree to which the midlevel mesocyclone and regions of near-surface circulation are well aligned appears to be at least as fundamental as the magnitude of the dynamic forcing (in terms of the VPPGF) produced by the mesocyclone, at least for the narrow set of experiments considered here.

Overall, these conclusions suggest that a primary consideration when diagnosing the impact of the lowlevel wind profile on supercell tornadoes should be the degree to which the storm environment promotes the favorable alignment of near-surface circulation and dynamic forcing provided by the mesocyclone aloft. It is important to note that this does not necessarily negate the importance of other considerations, such as the strength of the dynamic forcing, which may be modulated by the amount of SRH in a given low-level wind profile, or the degree to which antecedent near-surface circulation is produced in a supercell. Rather, our conclusions suggest that favorable alignment is a necessary but not sufficient condition for near-surface vertical vorticity intensification.

Finally, we caution the reader that our simulations are over a very narrow range of highly idealized environmental conditions, such that particular orientations, depths, or magnitudes of low-level shear favorable for near-surface rotation herein may not be ideal for a different deep-layer wind profile or different thermodynamic conditions. Moreover, the inclusion of surface drag might also alter the degree to which outflow is restrained or surges relative to the mesocyclone. Thus, in the future, we hope to expand this work by comparing the influence of the low-level wind profile with varied thermodynamic soundings, where precipitable water, CAPE, and LCL are modified. Future studies will also measure the mesocyclone separation distance using radar measurements of a climatology of observed tornadic and nontornadic supercells in an effort to verify the findings in this modeling study.

Acknowledgments. The authors wish to thank Dr. George Bryan for supplying and maintaining the code for CM1 and Dr. Ryan Hastings for providing his pressure decomposition code. We also thank three anonymous reviewers for their comments, from which this manuscript has greatly benefited. This work is funded by NSF Grant AGS-1446342 through the NSF Physical and Dynamic Meteorology program. 


\section{REFERENCES}

Brooks, H. E., C. A. Doswell III, and R. B. Wilhelmson, 1994: The role of midtropospheric winds in the evolution and maintenance of low-level mesocyclones. Mon. Wea. Rev., 122, 126-136, https://doi.org/10.1175/1520-0493(1994)122<0126: TROMWI $>2.0 . \mathrm{CO} ; 2$.

Browning, K. A., 1964: Airflow and precipitation trajectories within severe local storms which travel to the right of the winds. J. Atmos. Sci., 21, 634-639, https://doi.org/10.1175/ 1520-0469(1964)021<0634:AAPTWS >2.0.CO;2.

Bryan, G. H., 2002: An investigation of the convective region of numerically simulated squall lines. Ph.D. thesis, The Pennsylvania State University, $181 \mathrm{pp}$.

— nonhydrostatic numerical models. Mon. Wea. Rev., 130, 2917-2928, https://doi.org/10.1175/1520-0493(2002)130<2917: ABSFMN $>2.0 . \mathrm{CO} ; 2$.

Bunkers, M. J., B. A. Klimowski, J. W. Zeitler, R. L. Thompson, and M. L. Weisman, 2000: Predicting supercell motion using a new hodograph technique. Wea. Forecasting, 15, 61-79, https://doi.org/10.1175/1520-0434(2000)015<0061: PSMUAN $>2.0 . \mathrm{CO} ; 2$.

Coffer, B., and M. D. Parker, 2015: Impacts of increasing low-level shear on supercells during the early evening transition. Mon. Wea. Rev., 143, 1945-1969, https://doi.org/10.1175/MWR-D-14-00328.1.

— tornadic VORTEX2 environments. Mon. Wea. Rev., 145, 149-180, https://doi.org/10.1175/MWR-D-16-0226.1.

Craven, J. P., and H. E. Brooks, 2004: Baseline climatology of sounding derived parameters associated with deep, moist convection. Natl. Wea. Dig., 28, 13-24.

Davies-Jones, R., 1984: Streamwise vorticity: The origin of updraft rotation in supercell thunderstorms. J. Atmos. Sci., 41, 2991-3006, https://doi.org/10.1175/1520-0469(1984)041<2991: SVTOOU $>2.0 . \mathrm{CO} ; 2$.

Deardorff, J. W., 1980: Stratocumulus-capped mixed layers derived from a three-dimensional model. Bound.-Layer Meteor., 18, 495-527, https://doi.org/10.1007/BF00119502.

Dowell, D. C., and H. B. Bluestein, 2002: The 8 June 1995 McLean, Texas, storm. Part II: Cyclic tornado formation, maintenance, and dissipation. Mon. Wea. Rev., 130, 2649-2670, https://doi.org/ 10.1175/1520-0493(2002)130<2649:TJMTSP>2.0.CO;2.

Esterheld, J. M., and D. J. Guiliano, 2008: Discriminating between tornadic and nontornadic supercells: A new hodograph technique. Electron. J. Severe Storms Meteor., 3 (2), http:// www.ejssm.org/ojs/index.php/ejssm/issue/view/13.

Frame, J. W., and P. M. Markowski, 2013: The dynamical influences of anvil shading on simulated supercell thunderstorms. Mon. Wea. Rev., 141, 2802-2820, https://doi.org/ 10.1175/MWR-D-12-00146.1.

Gilmore, M. S., and L. J. Wicker, 1998: The influence of midtropospheric dryness on supercell morphology and evolution. Mon. Wea. Rev., 126, 943-958, https://doi.org/10.1175/ 1520-0493(1998)126<0943:TIOMDO > 2.0.CO;2.

Grzych, M. L., B. D. Lee, and C. A. Finley, 2007: Thermodynamic analysis of supercell rear-flank downdrafts from Project ANSWERS. Mon. Wea. Rev., 135, 240-246, https://doi.org/ 10.1175/MWR3288.1.

Hastings, R., and Y. Richardson, 2016: Long-term morphological changes in simulated supercells following mergers with nascent supercells in directionally varying shear. Mon. Wea. Rev., 144, 471-499, https://doi.org/10.1175/MWR-D-15-0193.1.
Hirth, B. D., J. L. Schroeder, and C. C. Weiss, 2008: Surface analysis of the rear-flank downdraft outflow in two tornadic supercells. Mon. Wea. Rev., 136, 2344-2363, https://doi.org/ 10.1175/2007MWR2285.1.

Houston, A. L., 2016: The sensitivity of deep ascent of cold-pool air to vertical shear and cold-pool buoyancy. Electron. J. Severe Storms Meteor., 11 (3), http://www.ejssm.org/ojs/index.php/ ejssm/article/viewArticle/151.

Kis, A. K., and J. Straka, 2010: New quantification of hodograph shape in nocturnal tornadic environments and its application to forecasting. 25th Conf. on Severe Local Storms, Denver, CO, Amer. Meteor. Soc., P6.8, https://ams.confex.com/ams/ 25SLS/techprogram/paper_176078.htm.

Lemon, L. R., and C. A. Doswell, 1979: Severe thunderstorm evolution and mesocyclone structure as related to tornadogenesis. Mon. Wea. Rev., 107, 1184-1197, https://doi.org/ 10.1175/1520-0493(1979)107<1184:STEAMS>2.0.CO;2.

Leslie, L. M., and R. K. Smith, 1978: The effect of vertical stability on tornadogenesis. J. Atmos. Sci., 35, 1281-1288, https://doi. org/10.1175/1520-0469(1978)035<1281:TEOVSO > 2.0.CO;2.

Markowski, P. M., and Y. P. Richardson, 2009: Tornadogenesis: Our current understanding, forecasting considerations, and questions to guide future research. Atmos. Res., 93, 3-10, https://doi.org/10.1016/j.atmosres.2008.09.015.

- and —, 2014: The influence of environmental low-level shear and cold pools on tornadogenesis: Insights from idealized simulations. J. Atmos. Sci., 71, 243-275, https://doi.org/ 10.1175/JAS-D-13-0159.1.

$\longrightarrow$, and — 2017: Large sensitivity of near-surface vertical vorticity development to heat sink location in idealized simulations of supercell-like storms. J. Atmos. Sci., 74, 1095-1104, https://doi.org/10.1175/JAS-D-16-0372.1.

_ , J. M. Straka, and E. N. Rasmussen, 2002: Direct surface thermodynamic observations within the rear-flank downdrafts of nontornadic and tornadic supercells. Mon. Wea. Rev., 130, 1692-1721, https://doi.org/10.1175/1520-0493(2002)130<1692: DSTOWT $>2.0$.CO;2.

C. Hannon, J. Frame, E. Lancaster, A. Pietrycha, R. Edwards, and R. Thompson, 2003a: Characteristics of vertical wind profiles near supercells obtained from the Rapid Update Cycle. Wea. Forecasting, 18, 1262-1272, https://doi. org/10.1175/1520-0434(2003)018<1262:COVWPN $>2.0$. CO; 2 .

— J. M. Straka, and E. N. Rasmussen, 2003b: Tornadogenesis resulting from the transport of circulation by a downdraft: Idealized numerical model simulations. J. Atmos. Sci., 60, 795-823, https://doi.org/10.1175/1520-0469(2003)060<0795: TRFTTO $>2.0 . \mathrm{CO} ; 2$.

, E. Rasmussen, J. Straka, R. Davies-Jones, Y. Richardson, and R. J. Trapp, 2008: Vortex lines within low-level mesocyclones obtained from pseudo-dual-Doppler radar observations. Mon. Wea. Rev., 136, 3513-3535, https://doi.org/10.1175/ 2008MWR2315.1.

Marquis, J., Y. Richardson, P. Markowski, D. Dowell, and J. Wurman, 2012: Tornado maintenance investigated with high-resolution dual-Doppler and EnKF analysis. Mon. Wea. Rev., 140, 3-27, https://doi.org/10.1175/MWR-D-11-00025.1.

Miller, D. J., 2006: Observations of low-level thermodynamic and wind shear profiles on significant tornado days. $23 \mathrm{rd}$ Conf. on Severe Local Storms, St. Louis, MO, Amer. Meteor. Soc., 3.1, https://ams.confex.com/ams/23SLS/techprogram/ paper_115403.htm.

Morrison, H., J. A. Curry, and V. I. Khvorostyanov, 2005: A new double-moment microphysics parameterization for 
application in cloud and climate models. Part I: Description. J. Atmos. Sci., 62, 1665-1677, https://doi.org/10.1175/JAS3446.1.

Nowotarski, C. J., and A. A. Jensen, 2013: Classifying proximity soundings with self-organizing maps toward improving supercell and tornado forecasting. Wea. Forecasting, 28, 783-801, https://doi.org/10.1175/WAF-D-12-00125.1.

Parker, M. D., 2014: Composite VORTEX2 supercell environments from near-storm soundings. Mon. Wea. Rev., 142, 508-529, https://doi.org/10.1175/MWR-D-13-00167.1.

Rasmussen, E. N., and D. O. Blanchard, 1998: A baseline climatology of sounding-derived supercell and tornado forecast parameters. Wea. Forecasting, 13, 1148-1164, https://doi.org/ 10.1175/1520-0434(1998)013<1148:ABCOSD > 2.0.CO;2.

Rotunno, R., and J. B. Klemp, 1982: The influence of shear-induced pressure gradient on thunderstorm motion. Mon. Wea. Rev., 110, 136-151, https://doi.org/10.1175/1520-0493(1982)110<0136: TIOTSI $>2.0 . \mathrm{CO} ; 2$

- and - 1985: On the rotation and propagation of simulated supercell thunderstorms. J. Atmos. Sci., 42, 271-292, https:// doi.org/10.1175/1520-0469(1985)042<0271:OTRAPO $>2.0$. $\mathrm{CO} ; 2$.

,$- \ldots$, and M. L. Weisman, 1988: A theory for strong, longlived squall lines. J. Atmos. Sci., 45, 463-485, https://doi.org/ 10.1175/1520-0469(1988)045<0463:ATFSLL > 2.0.CO;2.

Skinner, P. S., C. C. Weiss, M. M. French, H. B. Bluestein, P. M. Markowksi, and Y. P. Richardson, 2014: VORTEX2 observations of a low-level mesocyclone with multiple internal rear-flank downdraft momentum surges in the 18 May 2010 Dumas, Texas, supercell. Mon. Wea. Rev., 142, 2935-2960, https://doi.org/10.1175/MWR-D-13-00240.1.

Smith, B. T., R. L. Thompson, J. S. Grams, C. Broyles, and H. E. Brooks, 2012: Convective modes for significant severe thunderstorms in the contiguous United States. Part I: Storm classification and climatology. Wea. Forecasting, 27, 11141135, https://doi.org/10.1175/WAF-D-11-00115.1.

Snook, N., and M. Xue, 2008: Effects of microphysical drop size distribution on tornadogenesis in supercell thunderstorms.
Geophys. Res. Lett., 35, L24803, https://doi.org/10.1029/ 2008GL035866.

Thompson, R. L., R. Edwards, J. A. Hart, K. L. Elmore, and P. Markowski, 2003: Close proximity soundings within supercell environments obtained from the Rapid Update Cycle. Wea. Forecasting, 18, 1243-1261, https://doi.org/ 10.1175/1520-0434(2003)018<1243:CPSWSE $>2.0$. CO;2.

Trapp, R. J., G. J. Stumpf, and K. L. Manross, 2005: A reassessment of the percentage of tornadic mesocyclones. Wea. Forecasting, 20, 23-34, https://doi.org/10.1175/WAF-835.1.

Wakimoto, R. M., and B. E. Martner, 1992: Observations of a Colorado tornado. Part II: Combined photogrammetric and Doppler radar analysis. Mon. Wea. Rev., 120, 522-543, https://doi.org/10.1175/1520-0493(1992)120<0522: OOACTP $>2.0 . \mathrm{CO} ; 2$.

Weisman, M. L., and J. B. Klemp, 1982: The dependence of numerically simulated convective storms on vertical wind shear and buoyancy. Mon. Wea. Rev., 110, 504520, https://doi.org/10.1175/1520-0493(1982)110<0504: TDONSC $>2.0 . \mathrm{CO} ; 2$.

- and - 1984: The structure and classification of numerically simulated convective storms in directionally varying wind shears. Mon. Wea. Rev., 112, 2479-2498, https://doi.org/ 10.1175/1520-0493(1984)112<2479:TSACON>2.0.CO;2.

- and 1986: Characteristics of isolated convective storms. Mesoscale Meteorology and Forecasting, P. S. Ray, Ed., 331358, https://doi.org/10.1007/978-1-935704-20-1_15.

Wicker, L. J., 1996: The role of near-surface wind shear on lowlevel mesocyclone generation and tornadoes. Preprints, 18th Conf. on Severe Local Storms, San Francisco, CA, Amer. Meteor. Soc., 115-119.

Xu, Q., 1992: Density currents in shear flows-A two-fluid model. J. Atmos. Sci., 49, 511-524, https://doi.org/10.1175/ 1520-0469(1992)049<0511:DCISFA > 2.0.CO;2.

Xue, M., 2000: Density currents in two-layer shear flows. Quart. J. Roy. Meteor. Soc., 126, 1301-1320, https://doi.org/10.1002/ qj. 49712656506 . 